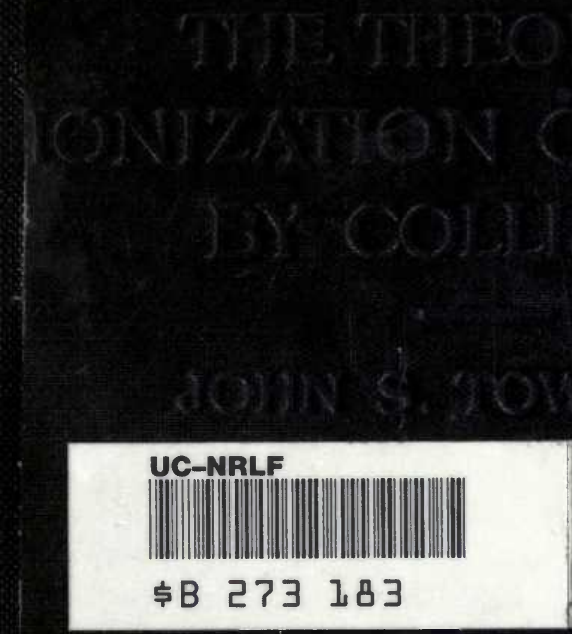

$$
\begin{aligned}
& 7 \\
& 0 \\
& 0 \\
& 0 \\
& 0
\end{aligned}
$$




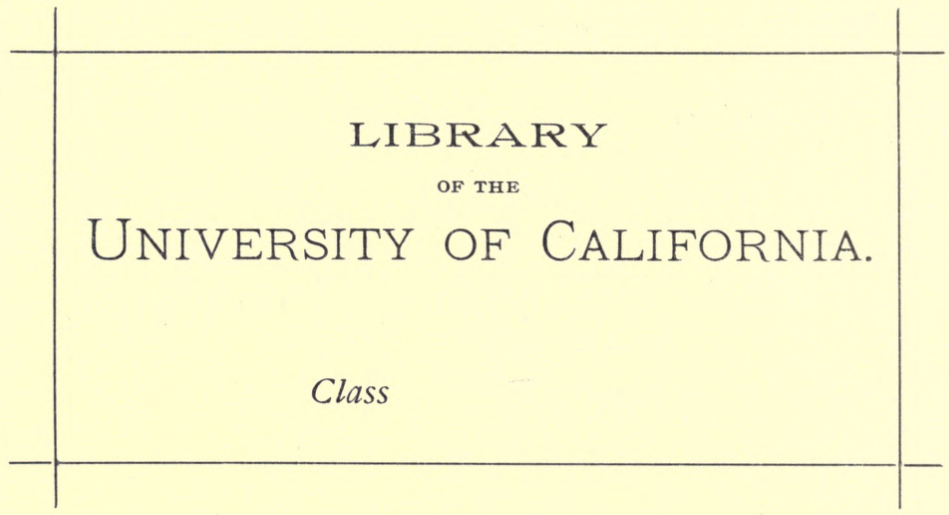






\section{THE THEORY OF IONIZATION OF GASES BY COLLISION}





\section{THE THEORY OF}

\section{IONIZATION OF GASES}

\section{BY COLLision}

\section{BY \\ JOHN S. TOWNSEND M.A. F.R.S.}

Wykeham Professor of Physics, Oxford,

Fellow of New Cot.lege, Oxford,

Formerly Fellow of Trinity College, Cambridge.

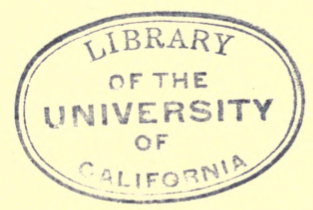

LONDON

CONSTABLE \& COMPANY LTD

10 Orange Street Leicester Square W C 
Q0702 $T 6$ 


\section{PREFACE}

AFTER studying the changes which take place in the conductivity of gases through which ions are passing under various conditions, I was led to propose the theory of ionization by collision to explain the development of currents in gases. The accuracy of the theory has been established by a large number of experiments specially arranged to measure conductivities which could be compared with the values obtained from theoretical considerations. The researches have brought to light the fact that ionization by collision takes place when comparatively small potential differences are established between electrodes in a gas at a suitable pressure, so that considerable multiplication of the ions may be obtained by this process with forty or fifty volts, and consequently with comparatively small velocities of the ions.

This is in marked contrast with the previously known cases of ionization produced by the motion of ions through a gas, where the velocity is always very high, sometimes approaching that of light. Such, for instance, is the case for the negative particles emitted by radioactive substances, or for the particles composing the cathode rays and the Lenard rays when the ions move with velocities that are acquired under the action of some thousands of volts.

The collision theory of the genesis of ions might have been framed from a consideration of some of Stoletow's 
experiments on ultra-violet light which were made in 1888, as considerable increases in the current of electricity through a gas were obtained with small voltages; but this method of explaining these experiments does not seem to have been suggested until (Nature, August 9, 1900) the theory had been substantially verified by the results of experiments on the conductivity produced by Röntgen rays. The explanation previously given of Stoletow's experiments was founded on a theory of electrical surface layers, but the fatal objection to it is that it fails to explain the fact that the current in the gas increases in geometric proportion as the distance between the plates increases in arithmetic proportion when the electric force remains constant. The agreement on the other hand between the experiments and the numbers calculated on the collision theory is so accurate that the phenomena are now attributed to the effects produced by collisions.

It is obvious from the large effects that may be obtained by the multiplication of ions with comparatively small electric forces that the process of ionization by collision is of fundamental importance in the development of large currents, and affords an explanation of many phenomena in connection with the discharge of electricity through gases. In particular, I may mention the application of these principles to account for sparking in gases. The exact value of the sparking potential, agreeing with the experimental determinations within a few per cent., may be calculated for a uniform field for different pressures of the gas, and the leading features of the more complicated phenomena obtained with large currents may also be accounted for.

The various points in connection with the theory which 
have been fairly well established by experimental methods have hitherto only appeared in different original papers, and those who are interested in the subject have found it difficult to get a general outline of the principles which are involved. The text-books that have been written on the conductivity of gases do not contain a good description of the methods by which the fundamental principles are established, and the attempts that have been made to explain well-known phenomena by the aid of the theory of collisions involve so many arbitrary assumptions, and are so inaccurate, that the results obtained are in many cases of no value.

It is in the hope of removing some of these difficulties that I have undertaken to write this account of the theory of ionization by collision and to discuss some of the phenomena in which the effects produced by collisions appear to play a predominant part.

In arranging this work for publication I have derived great advantage from suggestions that have been made to me by Mr. C. E. Haselfoot; my best thanks are due to him for the assistance he has thus rendered, and also for having undertaken to correct the proofs. 



\section{CONTENTS}

\section{CHAPTER I}

\section{Ionization By Negative Ions}

SECTION

1. Variation of current with electric force. . . . 1

2. Variation of current with distance between the electrodes when the force is constant . . . . 3

3. Determination of $a$ from experiments with ultra-violet light. . . . . . . . . . 5

4. Determination of $a$ from experiments with Röntgen

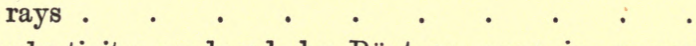

5. Conductivity produced by Röntgen rays in a gas between cylindrical electrodes . . . .

6. Negative ions generated by various methods have the same ionizing power . . . . . .

7. Comparison of the masses of negative ions in liquids and gases; corpuscular state . . . . . . 15 8. Representation of the values of $a$ by a single curve $\quad$. 17

9. Agreement between the experimental results and the

$$
\text { equation } \frac{a}{p}=f\left(\frac{\mathrm{X}}{p}\right) \text {. }
$$

10. Properties of the curves $\frac{\alpha}{p}=f\left(\frac{X}{p}\right)$. . . .

11. Maximum value of $a$; mean free path of negative ions. . . . . . . . . . .

12. Comparison of the values of $\alpha$ with the expression $\mathrm{Ne}_{\epsilon}-\frac{\mathrm{NV}}{\mathrm{X}}$ 
13. Molecular dimensions deduced from the mean free paths of negative ions . . . . . . . $\quad$. 29

14. Application of the theory to Stoletow's experiments - 30

15. Determination of the pressure corresponding to the maximum value of $\alpha$ for a given force . . . 34

16. Comparison of the velocities of ions and molecules $\quad 36$

\section{CHAPTER II}

\section{IoNizAtion BY Positive IoNs}

17. Conductivity between parallel plates when positive and negative ions generate others by collisions . . 38

18. Agreement between experiments and theory . . 42

19. Curves representing $\beta / p$ as a function of $\mathrm{X} / p$; comparison of effects produced by positive and negative ions . . . . . . . . . .

\section{CHAPTER III}

Sparking Potential in a Uniform Electric Field

20. Description of phenomena accompanying discharges; sparking potential . . . . . . . . 51

21. Potential required to maintain a discharge . . . 53

22. Properties of pointed and cylindrical electrodes . . 54

23. Condition for sparking in a uniform electric field.

Tables giving the sparking potentials determined

theoretically and experimentally . . . . 55

24. Effects of initial ionization . . . . . . 60

CHAPTER IV

Theory of Electric Discharges in Fields of Force which ARE NOT UNIFORM.

25. Description of phenomena to be investigated

26. Condition for the maintenance of a current by effects of collisions in any field of force . . . . 
27. Currents accompanied by a positive charge in the gas .

28. Increase of force at cathode accompanied by decrease of potential required to maintain a current between the electrodes for pressures above the critical pressure . . . . . .

29. Simple experiments to illustrate the effect of concentrating the force near the cathode . . .

30. Comparison of the effects of concentrating the force near the cathode and near the anode; explanation of sparking potentials for positive and negative points

31. Phenomena at pressures below the critical pressure

32. Processes of ionization that may account for effects obtained at low pressures

33. Cathode fall of potential ; ionization in the space near the cathode when the cathode fall of potential is established

34. Sparking potential at atmospheric pressure for very short distances between the electrodes . .

35. Remarks on processes of ionization which account for

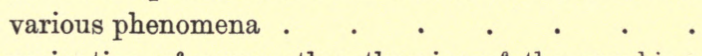

36. Examination of some other theories of the sparking potential . . . . . . . . . 



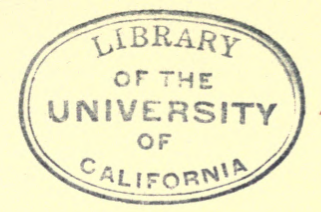

\title{
THE THEORY OF IONIZATION OF
}

\section{GASES BY COLLISION}

\author{
CHAPTER I \\ IONIZATION BY NEGATIVE IONS
}

1. Variation of current with electric force.

The process of ionization by collision between ions and molecules of a gas may be examined by investigating

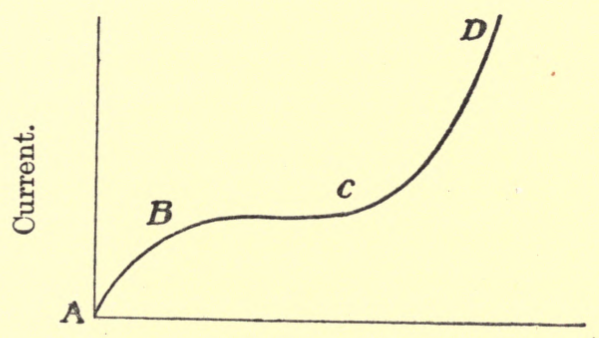

Electric Force.

Figure 1.

the currents between parallel plate electrodes when ultraviolet light falls on the negative electrode or when the gas is ionized by Röntgen rays. If the gas is at a high pressure, the current increases with the electric force and 
attains a maximum value, which is not exceeded unless very large forces are used. It is possible, however, by reducing the pressure of the gas, to make the ions travel with sufficient velocity to generate others by collisions with molecules, even when the potential differences employed are small, and thus with a few hundred volts to obtain large increases in the current. ${ }^{1}$ The curve, figure 1, showing the connection between the current and electric force in a gas at low pressure, may be taken as illustrating this effect.

In the first stage, A B, the current between the plates increases with the electro-motive force. The rate of increase diminishes as the force increases, and the current tends to attain a maximum value.

In the second stage, $\mathrm{B} \mathrm{C}$, the current remains practically constant and shows only small variations for large changes in the force. If the ions are produced by the action of Röntgen rays or Becquerel rays, the constant value is attained when the force is sufficiently great to collect all the positive and negative ions on the electrodes, but before this value is reached an appreciable number of the ions is lost by recombination. Again, the ions may be produced by the action of ultra-violet light on the negative electrode. In this case, if the force is too small, some of the ions do not reach the positive electrode, but diffuse through the gas to the negative electrode.

In the third stage, C D, when the force is still further increased, there is a large increase in the conductivity. This can be explained on the hypothesis that new ions are generated by collisions, at first practically by negative

1 Nature, Vol. lxii., August 9, 1900. 
ions alone, but as the force increases and the sparking potential is approached, the positive ions also acquire the property of producing others to an appreciable extent.

2. Variation of current with distance between the electrodes when the force is constant.

In the earlier experiments which were made to test the theory the initial ionization was produced by the action of Röntgen rays. The simplest conditions, however, are realized when the initial ionization consists of negative ions set free from a metal surface by a beam of ultra-violet light.

When the light falls on a metal plate a number $n_{0}$ of negative ions are set free which can be made to travel various distances through a gas under any required force to a parallel plate positively charged. If no new ions are produced by collisions the number reaching the positive plate will be $n_{0}$, and the current will be independent of the distance between the plates. If, however, the ions produce others by collisions with the molecules of the gas between the plates, the number reaching the positive plate will increase and will depend on the distance between the plates. In fact, if each negative ion set free from the metal plate produces $a$ new negative ions in going through a centimetre of the gas, and if the new ions produced in the gas have exactly the same property of generating others by collisions, then the number that arrive at the positive plate will be $n_{0} \epsilon^{a l}$ where $l$ is the distance between the plates. For let $n$ be number of ions produced in a layer of thickness, $x$, measured from the negative electrode, the number $n$ including the original 
$n_{0}$ ions. In passing through a path of length $d x$ these ions produce nadx new ions by collisions, so that $d n=n a d x$. This equation on integration gives $\log n=a x+$ constant, or $n=n_{0} \epsilon^{\alpha x}$ since $n_{0}$ is the value of $n$ corresponding to $x=0$.

The quantity a depends only on the electric force and pressure of the gas, and if these are constant the charges $n_{1}, n_{2}$, etc., acquired by the positive plate for different distances $l_{1}, l_{2}$, etc., between the plates will be

$$
n_{1}=n_{0} \epsilon^{a l_{1}} ; n_{2}=n_{0} \epsilon^{a l_{2}} \text {; etc. }
$$

Hence for equal increments of the distance the ratios of the successive charges will be the same, viz.-

$$
\frac{n_{2}}{n_{1}}=\frac{n_{3}}{n_{2}}=\text { etc. }=\epsilon^{a\left(l_{2}-l_{1}\right) .}
$$

The conditions specified above are easily realized in practice, and experiments show that this simple exponential law ${ }^{1}$ for the increase of the current with the distance $l$ is accurately true for small distances between the plates; for larger distances the conductivity rises more rapidly owing, as will be explained later, to the effect of positive ions; but for simplicity the currents between plates separated by short distances will be considered first.

1 These results showed definitely that it was necessary to abandon the older theories of surface layers by which these phenomena were formerly explained. Such theories provided, it is true, a possible explanation of the increase of current with increase of force, as it is conceivable that the greater the force the greater the number of ions drawn from the plate. But it is clear that if the force at the surface is kept constant an increase in the distance between the plates should not affect the current, and this is quite contrary to the experimental results. 
3. Determination of $a$ from experiments with ultra-violet light.

The principal parts of the apparatus which was used to find the conductivities are shown in figure 2. The currents take place in the gas between the plates $A$ and B. The upper plate from which the ions are set free was of zinc and was fixed to a micrometer screw for

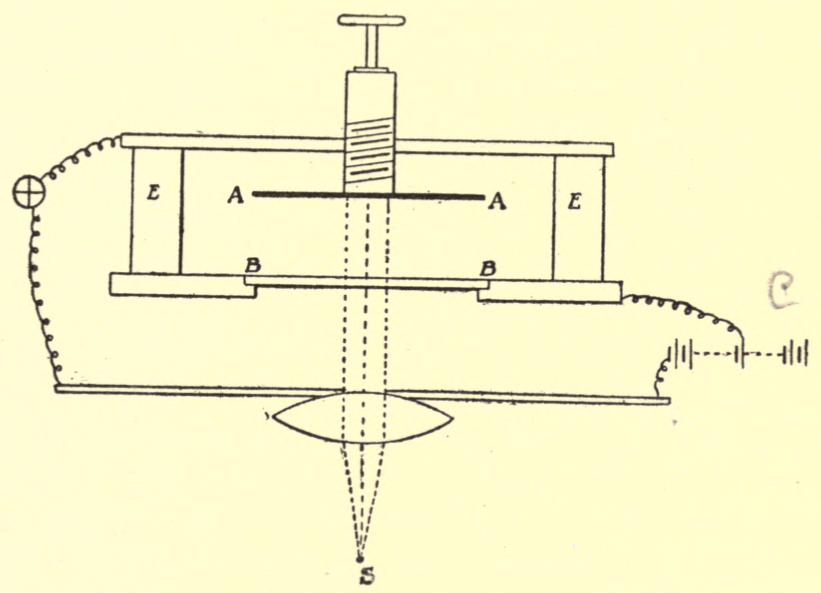

Figure 2.

adjusting the distance between the plates. Insulation was provided by the ebonite pillars $\mathrm{E}$. The lower plate B was of quartz silvered on the upper side so as to have a conducting surface in contact with the gas. A series of fine parallel lines were ruled on the quartz forming a transparent grating over a small area at the centre of the plate. The light from a spark gap $\mathrm{S}$ in a leyden jar circuit passed through a quartz lens and the grating, 
and falling on the upper plate set free negative ions from the zinc surface. The apparatus was covered with an air-tight glass cover, so that the pressure of the gas could be reduced, and suitable means were provided for turning the micrometer screw and making connection with the plates. The plate $\mathrm{B}$ was raised to any required potential by connecting it to a number of small accumulators. In a set of experiments in which it was necessary to maintain a uniform force, the number of cells used was proportional to the distance between the plates. When the currents passing between the plates are small the conductivities may be measured by a sensitive quadrant electrometer connected to the upper plate, but with large currents it is necessary to use an induction balance in order to get accurate determinations. ${ }^{1}$ The currents must in all cases be comparatively small so that the uniformity of field may not be disturbed by the electrostatic force produced by the separation of the. ions in the gas.

The following examples may be given of experiments with air at 2.5 millimetres pressure. With a force of 350 volts per centimetre, the currents for distances of 1,3 , and 5 millimetres between the plates were proportional to $1,2 \cdot 06$, and $4 \cdot 22$ respectively. For the same distances the currents were proportional to $1,4.24$, and 18.3 when the force was 525 volts per centimetre. The values of $a$ for the different forces may be obtained from the currents. Thus for 350 volts per centimetre the value of $a$ is given by the equation

$$
\epsilon^{\cdot 2 a}=\frac{2 \cdot 06}{1}=\frac{4 \cdot 22}{2 \cdot 06} \text {. }
$$

${ }^{1}$ See paper, Philosophical Magazine, November, 1903. 
4. Determination of a from experiments with Röntgen rays.

When the initial ionization is not produced at an electrode, but consists of positive and negative ions generated in the gas between the electrodes, as, for instance, by Röntgen rays or by Becquerel rays, the effect produced by collisions of the negative ions may be calculated as follows:-Let each negative ion produce $a$ others by collisions whilst passing through a centimetre of the gas, and let $n_{0}$ be the tctal number produced by the rays between the plates when at a distance $l$ apart. In the layer of thickness $d x$ at a distance $x$ from the positive electrode a number $\frac{n_{0} d x}{l}$ are generated by the rays, and in passing through the distance $x$ this number is increased to $\frac{n_{o} d x}{l} \times \epsilon^{a x}$ so that the total number of negative ions $n$ reaching the positive electrode is

$$
n=\int_{0}^{l} \frac{n_{0} \epsilon^{a x} d x}{l}=\frac{n_{0}\left(\epsilon^{a l}-1\right)}{a l} .
$$

The ratio $\frac{n}{n_{0}}$ may be obtained from the curve giving the connection between the current and the electric force, $n_{0}$ being the constant current when no new ions aro produced by collisions, and $n$, the current corresponding to a force $\mathrm{X}$. The value of $a$ for the force $\mathrm{X}$ is therefore given by the equation $\frac{n}{n_{0}}=\frac{\epsilon^{a l}-1}{a l}$. The accuracy of this formula has been tested experimentally, and it has been found that for a given force the value of $\frac{n}{n_{0}}$ varies with the distance $l$, but that the values of $a$ 
abtained for the different distances are the same within the limits of experimental error.

The curves, ${ }^{1}$ figures 3 and 4 , may be taken as examples. In each figure the curve corresponding to a distance of two centimetres between the plates rises more rapidly than that corresponding to one centimetre,

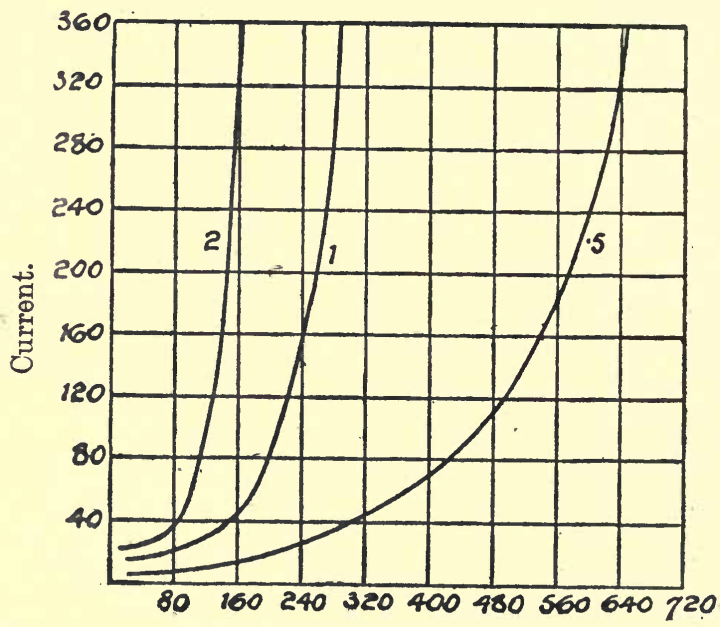

Volts per centimetre.

Current-electric force curves for air at $1.1 \mathrm{~mm}$. pressure, with distances of 2,1 and $\cdot 5 \mathrm{cms}$. between the plates.

Figure 3.

and in the curve corresponding to $\cdot 5$ centimetre the rate of increase with the force is much less than in either of the other two cases. It will be noticed that the curves are

1 The sets of curves at different pressures may be found in the paper Philosophical Magazine, February, 1901. 
practically parallel to the axis of $x$ for the low forces, so that $n_{0}$, the number of ions produced by the rays, is proportional to the currents for forces of 10 or 15 volts per centimetre. The following numbers may be taken as illustrations:

In the experiments at a pressure of 1.1 millimetre, and

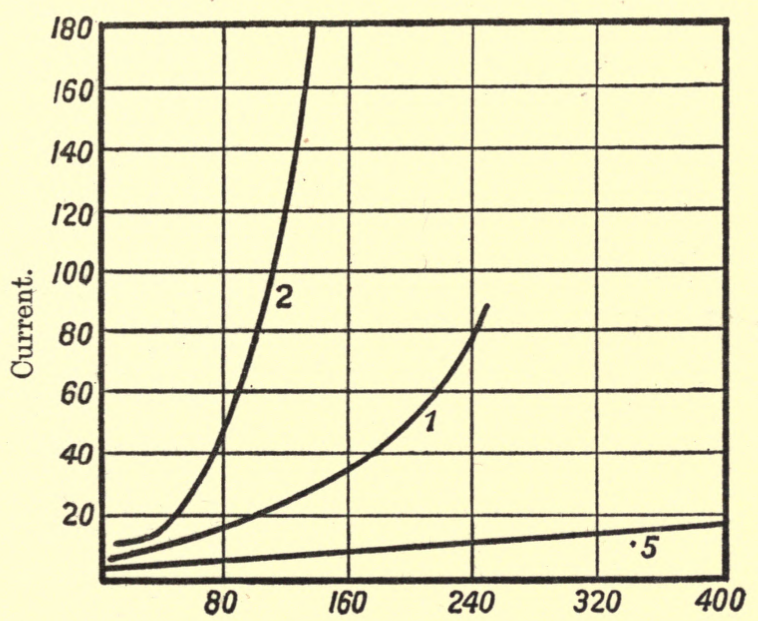

Volts per centimetre.

Current-electric force curves for air at $\cdot 385 \mathrm{~mm}$. pressure, with distances of 2,1 and $5 \check{c m s}$. between the plates.

\section{Figure 4.}

a force of 160 volts per centimetre the value of $a$ deduced from the curve, figure 3 , corresponding to a distance of 2 centimetres between the plates is $2 \cdot 02$, and from the second curve the value 1.98 was obtained. With the same force and a pressure of $\cdot 385$ millimetre $a$ increases, 
the values obtained from the curves, figure 4 , being $2: 8$, $2 \cdot 9$, and $2 \cdot 9$. Retaining the same force and making further reductions in the pressure, $a$ is found to diminish; its mean value deduced from similar experiments at 171 millimetre pressure being $2 \cdot 15$.

When the pressure is constant, $a$ increases with the force and approaches a maximum value, which is attained when the force is so great that a new pair of ions are formed at each collision. For the smaller forces $a$ has a smaller value since the velocity acquired by an ion along a free path is only large enough to produce others by collision in the case of the longer free paths.

When the force is constant and the pressure reduced, as in the experiments at 160 volts per centimetre which have been quoted, the value of a increases, attains a maximum, and finally diminishes again. This also agrees with the theory, for at high pressures the free paths are very short and the ions do not acquire a large velocity and do not ionize the molecules with which they collide. As the pressure is reduced the free paths are increased, so that along the longer paths a high velocity is attained. The proportion of collisions which result in new ions being formed thus increases, but the total number of collisions per centimetre diminishes. When the pressure is reduced beyond a certain point the values of $a$ begin to diminish, as the number of molecules with which an ion collides may become very small. The results of the experiments may thus be seen to be in general agreement with the theory, but the variations of $a$ obtained by altering the force and pressure will be examined more fully when the curves connecting $a, \mathrm{X}$, and $p$ are explained. 
5. Conductivity produced by Röntgen rays in a gas between cylindrical electrodes.

There is one point in connection with the currentelectric force curves obtained with Röntgen rays which remains to be decided by experiment. In this case both positive and negative ions are produced initially in the gas by the rays, so that it is not evident from the experiments with parallel plates that the results are due to negative ions. Obviously the effects might be due to positive ions if it happened that they had the property of generating others by collisions while the negative ions were inactive. It is, however, easy to show by using electrodes of different shapes that the effects must be attributed to negative ions. ${ }^{1}$ If the gas is contained in a spherical conductor which acts as one of the electrodes, the other electrode being a small sphere at the centre, or if a cylinder and a co-axial wire of small radius are used as electrodes, then for small differences of potential the current through the gas is the same in both directions and corresponds to the total number of ions generated by the rays; but when the difference of potential is large the currents are no longer equal. It has been found that when the outer electrode is negative and the electromotive force is increased a large increase in the current is obtained. In this case all the negative ions produced by the rays traverse the field of strong electric force in the neighbourhood of the small inner electrode, and acquire sufficient velocity to generate others by collisions with molecules of the gas. On the other hand, when the outer electrode is positive and similar

${ }^{1}$ See Nature, Vol. lxii., August 9, 1900. 
increases in electromotive force are made, the corresponding increases of current are very small. In this case the negative ions travel outwards, and only a few of those produced by the rays pass through the field of strong electric force, so that the number of new ions produced by collisions is comparatively small. Experiments

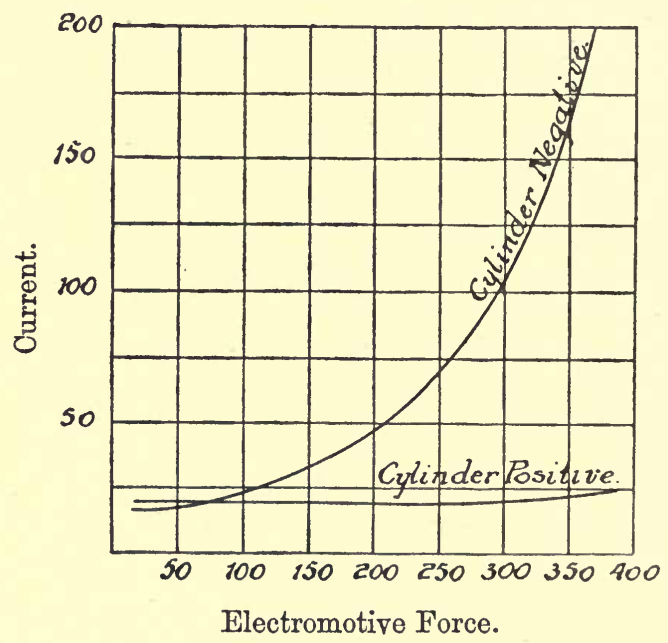

Figure 5.

on the currents between concentric cylinders have been made by Mr. Kirkby, which show in a striking manner the effect of changing the direction of the electric force. The curves, figure 5, for air at a pressure of 3.53 millimetres give the currents between a cylinder of $4 \cdot 15$ centimetres diameter and a concentric wire of 206 millimetres diameter when the air is ionized by Röntgen rays. $^{1}$

1 P. J. Kirkby, Philosophical Magazine, February, 1902. 
When the cylinder is negative new ions are produced as the force increases, so that when the potential difference between the wire and cylinder is 360 volts the current is ten times as great as that which is due to the ions that are produced by the direct action of the rays.

When the cylinder is positive there is no appreciable change in the current until the potential of the cylinder is more than 300 volts above the wire, and with 360 volts the current increases only by one-fifth of its initial value.

These results show that in the previous experiments with parallel plate electrodes the increase of conductivity must have been due to negative ions.

6. Negative ions generated by various methods have the same ionizing power.

Without considering the absolute values of $a$ that have been found, it is possible to deduce some interesting conclusions as to the properties of negative ions. Since $a$ is independent of the distance between the plates $l$, the new ions must generate others by collisions to exactly the same extent as the original ions by which they are themselves produced. From this it follows that the negative ions generated by collisions in any gas must in each case be the same as those produced by the external agencies.

Now when ultra-violet light is used, the ions set free from the zinc plate are the same for different gases; hence the negative ions produced by collisions from the molecules of different gases and vapours must be all the same, being identical with those set free from the negative electrode. The gases for which the values of $a$ have been determined by experiments in which the 
initial ionization was produced by ultra-violet light, are air, hydrogen, carbon dioxide, water vapour, hydrochloric acid, ${ }^{1}$ nitrogen, ${ }^{2}$ argon, ${ }^{3}$ and helium. ${ }^{3}$ A high degree of accuracy can be obtained by this method, and the experiments are simpler than those in which Röntgen rays are used to generate the initial ionization.

Again experiments with Röntgen rays have been made with air, ${ }^{4}$ hydrogen, and carbon dioxide, and it is found that the values of $a$ are the same over large ranges of pressure as those obtained for these gases with ultraviolet light. Thus under all variations of pressure and electric force the negative ions produced in gases, either by Röntgen rays or by collisions, follow the same changes in ionizing power as the negative ions set free from a metal plate by ultra-violet light. It may be concluded, therefore, that they are all identical, and this is supported by independent experiments, which show that the charges on negative ions produced by various methods are all equal to a fixed atomic charge. ${ }^{5}$

It is interesting to remark that the positive ions, although they have the same charge ${ }^{6}$ as the negative,

${ }^{1}$ See papers by the author, Philosophical Magazine, June, 1902, and April, 1903.

${ }^{2}$ H. E. Hurst, Philosophical Magazine, April, 1906.

${ }^{8}$ E. W. B. Gill and F. B. Pidduck, Philosophical Magazine, August, 1908.

${ }^{4}$ See papers by the author, Philosophical Magazine, February, 1901, and by the author and Mr. Kirkby, Philosophical Magazine, June, 1901.

${ }^{5}$ See papers on Diffusion of Ions, Philosophical Transactions, Vol. exciii., 1899, and Vol. cxcv., 1900, also papers in Vols. lxxx., lxxxi., Ixxxii. of the Proceedings of the Royal Society.

${ }^{6}$ Recent experiments show that in some cases positive ions have twice the atomic charge, but as the charges on positive ions generated by collisions have not yet been specially investigated, 
have very different physical properties in gases at low pressures. It will be seen from the results obtained below that the positive ions must acquire a very much larger kinetic energy than the negative ions before new ions can be produced by their collisions with molecules. This difference between the positive and negative ions shows that the condition that new ions should be generated by a collision is not determined by the kinetic energy of the colliding particle. Thus with equal kinetic energies the ionizing power of the negative ions is much greater than that of the positive, and this can only be attributed to their possessing a larger velocity, and, consequently, also a much smaller mass. Hence, since hydrogen is one of the gases which have been examined, it follows that the mass of the negative ion in any gas is less than that of the positive ion in hydrogen.

A particular instance of this result was previously established, in the case of negative ions set free from a metal by ultra-violet light. The ratio of the mass to the charge on the ion has been found, by Sir J. J. Thomson, by means of experiments on the effect of a magnetic field on the motion of the ion, ${ }^{1}$ and it was deduced that the mass of an ion set free by ultra-violet light was of the order $1 / 1000$ of the mass of a hydrogen atom.

7. Comparison of the masses of negative ions in liquids and gases; corpuscular state.

In this connection it is interesting to observe that it will be assumed for simplicity that they have single atomic charges. See papers by the author and Mr. C. E. Haselfoot, Proceedings of the Royal Society, A. Vols. Ixxx., lxxxi., 1908, and Vol. Ixxxii., 1909.

1 J. J. Thomson, Philosophical Magazine, December, 1899. 
the ions in liquids are quite different from the ions is gases, although they have the same charges. Thus, in a solution of hydrochloric acid, the chlorine atom (whicl is the constituent of the molecule with the larger mass has a negative charge and the hydrogen atom a positive charge. In the gaseous state, on the other hand, hydrochloric acid conducts at low pressures just as other gases, and the negative ion is associated with a mass which is small compared with that of the positive ion.

At first sight the conclusions to which the experiments on the effects of collisions lead as to the masses of the ions appear to be inconsistent with experiments on the velocities of the ions and the rates of diffusion, which show that at high pressures there is no great difference between the apparent masses of positive and negative ions. In these cases the ions move in the gas as if comparatively large masses were connected with the atomic charges, a fact deduced from determinations of the rates of diffusion, which show, for example, that the rate of diffusion of ions in hydrogen is small as compared with the rate of diffusion of carbonic acid into hydrogen. The result thus obtained may be explained on the supposition that each ion is accompanied by a group of molecules which are attracted by the strong electric field in the neighbourhood of the ion. The number of molecules of the gas which are so affected would be determined principally by the charge on the ion, so that there would not be any great difference between the positive and negative ions as they have the same charges. The groups of molecules would remain attached to the ions when they are not moving with large velocities, but when large forces are acting and the ions move with large velocities in the gas at low pressure, the effect of the 
charge in attracting a molecule would only last for a short interval, so that for large values of $\mathrm{X} / p$ the ions would move with their own proper masses. The transition from one state to the other takes place gradually as the force rises, and it is probable in the case of the negative ions that the corpuscular state prevails even when the force is not large enough to cause new ions to be produced by collisions. One result which points to this conclusion is that when $\mathrm{X}$ is small there is no very rapid diminution of $a$ with the force as might be expected if the mass of the negative ion tended to increase.

Other experiments show also that the mass of the negative ion is much less than that of the positive ion when the forces acting are too small to produce ions by collision. Thus experiments on diffusion ${ }^{1}$ of ions in dry air at low pressure show that when $\mathrm{X} / p=\cdot 09$, the mass of the negative ion is much less than that of the positive ion, whereas even for $\mathrm{X}=20$ and $p=1$, the value of $a$ is inappreciable and only attains the value $\cdot 12$ when $\mathrm{X}=60$.

\section{Representation of the values of $a$ by a single curve.}

In order to examine the values of $a$ for different forces and pressures, it is convenient to present the experimental results in a special way by means of a curve for each gas. The method leads to great simplification as the value of $\alpha$ corresponding to any force and pressure may be obtained immediately from the curve. If $a$ be determined for a force $\mathrm{X}$ and pressure $p$, and the points whose co-ordinates are $a / p$ and $\mathrm{X} / p$ be marked on $a$ diagram, it will be found that they all lie on the same curve,

1 Proceedings of the Royal Society, 1908, Vol. Ixxxi., p. 470. 
or if the experiments be examined it will be noticed that if $a^{\prime}$ corresponds to a force $\mathrm{X}^{\prime}$ and a pressure $p^{\prime}$, then at a pressure $z p^{\prime}$, the value of $a$ will $z a^{\prime}$ when the force is $z \mathrm{X}^{\prime}, z$ being any multiplier.

In the later experiments the pressures were chosen so as to show this by inspection. ${ }^{1}$ Thus, with air at a pressure of 1 millimetre and a force of 350 volts per centimetre, $a=5.25$; at 2 millimetres pressure and with a force of 700 volts per centimetre, $a=10.5$. Another example may be taken from the experiments with hydrogen :

$$
\begin{aligned}
& p=8 \mathrm{~mm} ., \mathrm{X}=1050 \text { volts per cm., } a=14 \cdot 8 \\
& p=4 \mathrm{~mm} ., \mathrm{X}=525 \text { volts per cm., } a=7 \cdot 4 \\
& p=2 \mathrm{~mm} ., \mathrm{X}=262 \text { volts per cm., } a=3 \cdot 7
\end{aligned}
$$

The results of all the experiments for any one gas can therefore be recorded by a table of values of $a / p$ and $\mathrm{X} / p$ or by means of a curve ${ }^{2}$ representing one of these ratios as a function of the other $\frac{a}{p}=f\left(\frac{\mathrm{X}}{p}\right)$.

9. Agreement between the experimental results and

$$
\text { the equation } \frac{a}{p}=f\left(\frac{\mathrm{X}}{p}\right) \text {. }
$$

It is easy to see that the theory requires that this relation should exist between the variables $a, \mathrm{X}$, and $p$.

In passing through a centimetre in a gas an ion traverses free paths of various lengths between the collisions. The chance of producing a new ion by collision will depend on the velocity at impact, and this

1 Many examples may be found in the researches published in the Philosophical Magazine, November, 1903, and December, 1904.

2 This result was first obtained from the experiments with Röntgen rays. See Philosophical Magazine, February, 1901. 
is determined by the force $\mathrm{X}$ and the length of the path which is terminated by the collision. The lengths of the free paths are inversely proportional to the pressure, so that if the pressure is increased from $p$ to $z p$ all the free paths will be reduced to $1 / z$ of their original value. If the force $\mathrm{X}$ remained unaltered, the velocities on

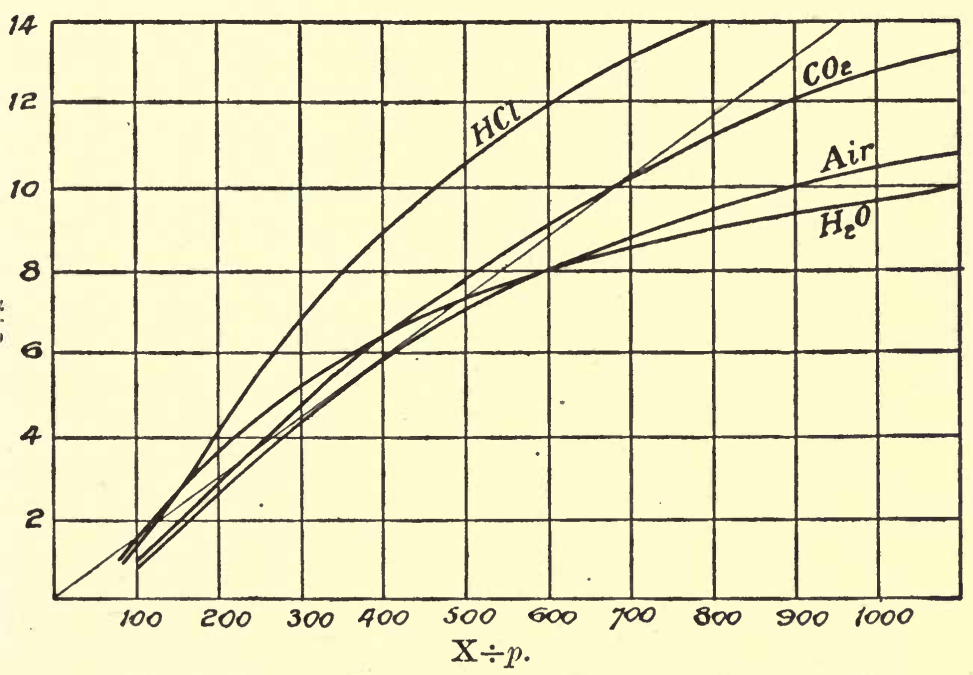

( $\mathrm{X}$ in volts per centimetre, $p$ in millimetres of mercury.)

Figure 6.

collision would be reduced, but if the force is increased to $z \mathrm{X}$ the velocities will be restored to their original values and the number of ions arising from a given number of collisions will be the same as before. Since the total number of collisions per centimetre is increased in the same ratio as the pressure, the value of $a$ will be increased to $z a$, when $\mathrm{X}$ and $p$ become $z \mathrm{X}$ and 
$z p$ respectively. Hence the three variables must be connected by an equation of the form $\frac{a}{p}=f\left(\frac{\mathrm{X}}{p}\right)$. The experiments confirm this result very accurately for all the gases, and so afford an important verification of the theory.

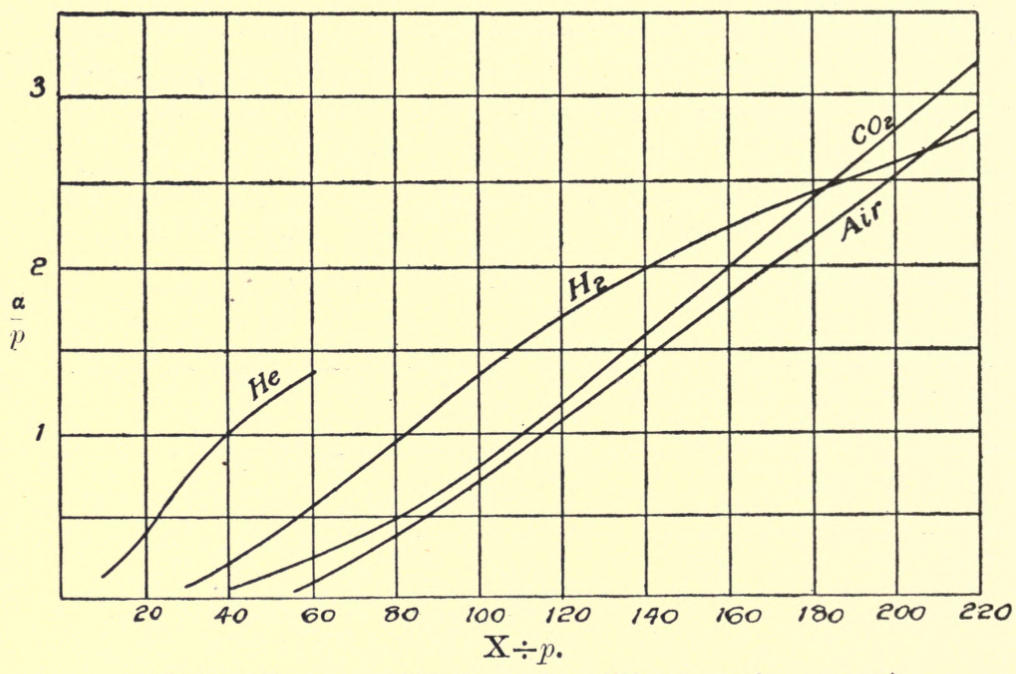

( $\mathrm{X}$ in volts per centimetre, $p$ in millimetres of mercury.)

Figure 7 .

The curves representing the values of $a / p$ corresponding to some of the larger values of $\mathrm{X} / p$ are given in Figs. 6 and 7.

The values of $a / p$ for the smaller values of $\mathrm{X} / p$ are given in Fig. 8 for some of the gases. 
10. Properties of the curves $\frac{a}{p}=f\left(\frac{\mathrm{X}}{p}\right)$.

The properties of the curves are in general agreement with the indications of the theory. For simplicity the pressure may be taken as unity which has been fixed as the pressure due to 1 millimetre of mercury. The

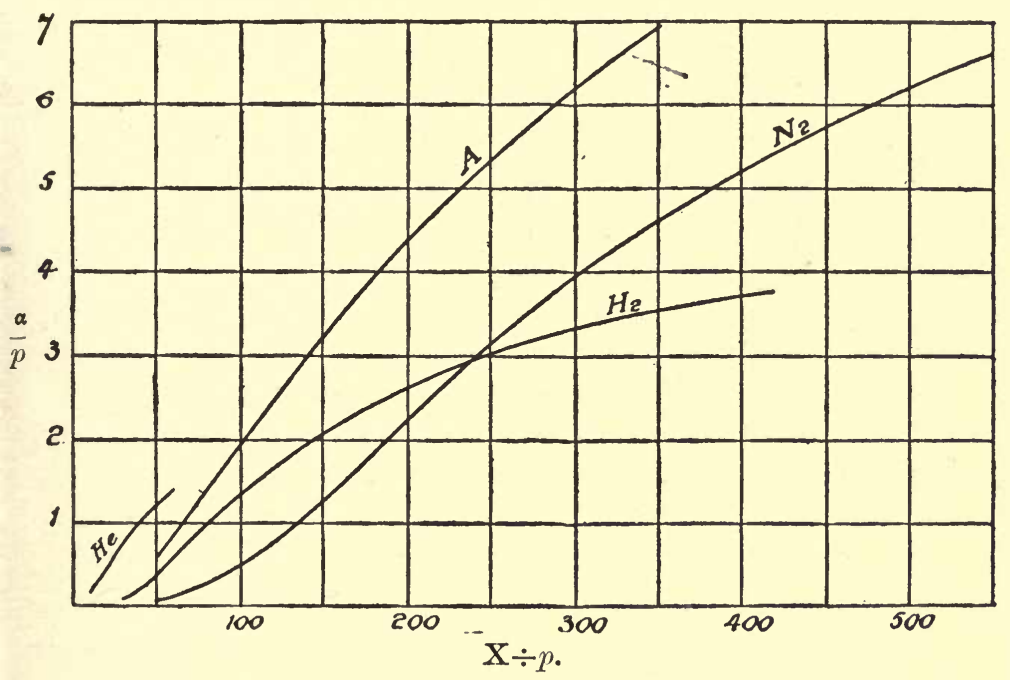

( $\mathrm{X}$ in volts per centimetre, $p$ in millimetres of mercury.)

Figure 8.

co-ordinates of any point then represent the electric force $\mathrm{X}$ and the number $a$ of molecules of the gas at a millimetre pressure that are ionized by a single ion in moving through one centimetre under this force. For small forces the quantity a practically vanishes; the ions in this case do not acquire sufficient velocity along any of their free paths to generate others by collisions. 
As the force increases a acquires a small value and subsequently increases still further, the ions during these stages acquiring sufficient velocity along some of their free paths to produce others by collisions. With the smaller forces such velocity is acquired only along the longer free paths, but with large forces the number of paths along which an ion gains this velocity becomes more numerous. When a becomes large its rate of increase with the force diminishes, and finally $a$ approaches a maximum value which should be attained when a new pair of ions is produced at each collision.

\section{Maximum value of $a$; mean free path of negative ions.}

The maximum value cannot be obtained experimentally by these methods, as sparking would ensue when large forces are used, unless the amount of gas between the electrodes is very small, and in this case the calculations would be inaccurate, as the distance between the plates would not be large compared with the free paths. The maximum values of $a$ for a pressure of 1 millimetre may nevertheless be deduced from the curves by the following method.

Let it be assumed that one pair of ions is formed when the velocity $u$ at collision exceeds a certain value. Also let it be supposed that the velocity of a negative ion is so much reduced by collision with a molecule that it practically starts from rest along its new path. An ion will then acquire the requisite velocity under the force $\mathrm{X}$ when it travels freely along a distance $y$ such that

$$
e \mathrm{X} y>\frac{m u^{2}}{2}
$$


or when Xy exceeds a certain potential V. The value of $a$ corresponding to $\mathrm{X}$ represents, therefore, the number of free paths per centimetre which exceed the value $y=\mathrm{V} / \mathrm{X}$. It is easy to express $a$ as a function of $\mathrm{X}$ and the mean free path. Let $\mathrm{N}$ be the total number of collisions that an ion makes with molecules in travelling through a distance of 1 centimetre in a gas at 1 millimetre pressure, so that $\frac{1}{\mathrm{~N}}$ is the mean free path. Let $n$ be the number of paths per centimetre which exceed the distance $y$. In going through the element $d y$ of these paths the number of collisions will be proportional to $n d y$, hence $-d n=k n d y$ where $k$ is a constant. On integrating, this equation gives $n=c \epsilon^{-k y}$, the constant $c$ being equal to $\mathrm{N}$, which represents the number of paths which exceed the value $y=0$. The constant $k$ may be found in terms of the mean free path, for since $-y d n$ is the sum of the paths whose lengths are between $y$ and $y+d y$, the sum of all the paths in 1 centimetre is

$$
-\int_{\mathrm{N}}^{0} y d n=\int_{0}^{\infty} \mathrm{N} k y \epsilon-k y d y=1,
$$

which gives $k=\mathrm{N}$.

Hence the number of paths per centimetre which exceed the distance $y$ is $\mathrm{N} \epsilon^{-\mathrm{N} y}$, which is therefore the value of $a$ when $y=\frac{\mathrm{V}}{\mathrm{X}}$.

The connection, therefore, between $a$ and $\mathrm{X}$ when $p=1$ is $a=\mathrm{N} \epsilon-\frac{\mathrm{NV}}{\mathrm{X}}$ (or in general $\left.\frac{a}{p}=\mathrm{N} \epsilon-\frac{\mathrm{NV} p}{\mathrm{X}}\right)$ where $\mathrm{N}$, the maximum value of $a$, is the number of collisions that an ion makes in travelling a distance of 1 
centimetre in the gas at 1 millimetre pressure, and the constant $\mathrm{V}$ represents the potential difference between the ends of a path along which an ion acquires sufficient velocity to generate others by collisions.

12. Comparison of the values of $a$ with the

$$
\text { expression } \mathrm{N} \epsilon-\frac{\mathrm{NV}}{\mathrm{X}} \text {. }
$$

The following tables give values of $a$ for the gases at 1 millimetre pressure, the forces $\mathrm{X}$ being expressed in volts per centimetre, and also the values of the quantity $\mathrm{N} \epsilon-\frac{\mathrm{NV}}{\mathrm{X}}$, the constants $\mathrm{N}$ and $\mathrm{V}$ having been chosen so that the formula should agree with the experimental results for the larger forces.

The values of a corresponding to any other pressure may be obtained by taking the numbers to indicate $\mathrm{X} / p$ and $a / p$ instead of $\mathrm{X}$ and $a$ respectively.

TABLE I.

AIR. $-\mathrm{N}=14 \cdot 6 ; \mathrm{V}=25^{\circ} \cdot 0$.

\begin{tabular}{|c|c|c|c|c|c|c|c|c|c|}
\hline $\mathrm{X}$. & 1000 & 800 & 700 & 600 & 500 & 400 & 300 & 200 & 100 \\
\hline $\begin{array}{c}a \text { found } \\
\text { experimentally. }\end{array}$ & $10 \cdot 5$ & $9 \cdot 3$ & $8 \cdot 7$ & $7 \cdot 9$ & $7 \cdot 0$ & $5 \cdot 82$ & $4 \cdot 4$ & $2 \cdot 6$ & $\cdot 72$ \\
\begin{tabular}{c}
$\mathrm{N} \epsilon-\frac{\mathrm{NV}}{\mathrm{X}}$ \\
\hline
\end{tabular} & $10 \cdot 1$ & $9 \cdot 3$ & $8 \cdot 6$ & $7 \cdot 9$ & $7 \cdot 0$ & $5 \cdot 85$ & $4 \cdot 3$ & $2 \cdot 34$ & $\cdot 38$ \\
\hline
\end{tabular}


Ionization by Negative Ions

TABLE II.

Nitrogen. $-\mathrm{N}=12 \cdot 4 ; \mathrm{V}=27 \cdot 6$.

\begin{tabular}{|c|c|c|c|c|c|c|}
\hline $\mathrm{X}$. & 600 & 500 & 400 & 300 & 200 & 100 \\
\hline $\begin{array}{c}\text { a found } \\
\text { experimentally. }\end{array}$ & $7 \cdot 0$ & $6 \cdot 2$ & $5 \cdot 2$ & $3 \cdot 95$ & $2 \cdot 3$ & $\cdot 42$ \\
$\mathrm{~N}_{\epsilon}-\frac{\mathrm{NV}}{\mathrm{X}}$ & $7 \cdot 0$ & $6 \cdot 25$ & $5 \cdot 3$ & 3.95 & $2 \cdot 24$ & $\cdot 41$ \\
\hline
\end{tabular}

TABLE III.

Hydrogen.-N=5.0; V=26.

\begin{tabular}{|c|c|c|c|c|c|c|}
\hline X. & 400 & 300 & 200 & 100 & 50 & 30 \\
\hline $\begin{array}{c}\alpha \text { found } \\
\text { experimentally. }\end{array}$ & $3 \cdot 7$ & $3 \cdot 3$ & $2 \cdot 62$ & $1 \cdot 36$ & $\cdot 36$ & $\cdot 08$ \\
\hline$N_{\epsilon}-\frac{N V}{X}$ & $3 \cdot 64$ & $3 \cdot 24$ & $2 \cdot 62$ & $1 \cdot 3 \tilde{\Sigma}$ & 35 & $\cdot 066$ \\
\hline
\end{tabular}

TABLe IV.

Carbon Dioxide. $-\mathrm{N}=20 ; \mathrm{V}=23 \cdot 3$.

\begin{tabular}{|c|c|c|c|c|c|c|c|c|c|c|}
\hline $\mathrm{X}$. & 1200 & 1000 & 800 & 700 & 600 & 500 & 400 & 300 & 200 & 100 \\
\hline $\begin{array}{c}\alpha \text { found } \\
\text { experimentally. } \\
\mathrm{N}_{\epsilon}^{-\frac{\mathrm{NV}}{\mathrm{X}}}\end{array}$ & $13 \cdot 7$ & $12 \cdot 6$ & $11 \cdot 0$ & $10 \cdot 2$ & $9 \cdot 1$ & $7 \cdot 8$ & $6 \cdot 4$ & $4 \cdot 8$ & $2 \cdot 8$ & $\cdot 82$ \\
\hline
\end{tabular}




\section{TABLE V.}

HYdRochloRIC ACID.-N=22.2; V=16.5.

\begin{tabular}{|c|c|c|c|c|c|c|c|c|c|c|}
\hline $\mathrm{X}$. & 1500 & 1000 & 800 & 700 & 600 & 500 & 400 & 300 & 200 & 100 \\
\hline $\begin{array}{c}\alpha \text { found } \\
\text { experimentally. }\end{array}$ & $17 \cdot 5$ & $15 \cdot 4$ & $14 \cdot 0$ & $13 \cdot 0$ & 11.9 & $10 \cdot 5$ & $8 \cdot 9$ & $6 \cdot 8$ & $4 \cdot 1$ & $1 \cdot 21$ \\
$\mathrm{~N}_{\epsilon}^{-\frac{\mathrm{NV}}{\mathrm{X}}}$ & $17 \cdot 5$ & $15 \cdot 4$ & $14 \cdot 0$ & $13 \cdot 2$ & $12 \cdot 0$ & $10 \cdot 65$ & $8 \cdot 9$ & 6.5 & 3.5 & .57 \\
\hline
\end{tabular}

\section{TABLe VI.}

WATER VAPOUR.-N=12.9; V $=22 \cdot 4$.

\begin{tabular}{|c|c|c|c|c|c|c|c|c|c|c|}
\hline $\mathrm{X}$. & 1000 & 900 & 800 & 700 & 600 & 500 & 400 & 300 & 200 & 100 \\
\hline $\begin{array}{c}\alpha \text { found } \\
\text { experimentally. }\end{array}$ & $9 \cdot 7$ & $9 \cdot 4$ & $9 \cdot 0$ & $8 \cdot 5$ & $7 \cdot 95$ & $7 \cdot 2$ & $6 \cdot 35$ & $5 \cdot 2$ & $3 \cdot 6$ & $1 \cdot 31$ \\
$\mathrm{~N} \epsilon^{-\frac{\mathrm{NV}}{\mathrm{X}}}$ & $9 \cdot 7$ & $9 \cdot 35$ & $8 \cdot 9$ & $8 \cdot 54$ & $7 \cdot 96$ & $7 \cdot 2$ & $6 \cdot 25$ & $4 \cdot 9$ & $3 \cdot 0$ & $\cdot 71$ \\
\hline
\end{tabular}

TABLE VII.

Argon. $-\mathrm{N}=13 \cdot 6 ; \mathrm{V}=17 \cdot 3$.

\begin{tabular}{|c|c|c|c|c|c|c|c|}
\hline $\mathrm{X}$. & 600 & 500 & 400 & 300 & 200 & 100 & 50 \\
\hline $\begin{array}{c}\text { a found } \\
\text { experimentally. }\end{array}$ & $9 \cdot 2$ & $8 \cdot 5$ & $7 \cdot 5$ & $6 \cdot 2$ & $4 \cdot 4$ & $2 \cdot 0$ & $\cdot 58$ \\
$\mathrm{N \epsilon}-\frac{\mathrm{NV}}{\mathrm{X}}$ & $9 \cdot 2$ & $8 \cdot 5$ & $7 \cdot 5$ & $6 \cdot 2$ & $4 \cdot 2$ & 1.3 & $\cdot 12$ \\
\hline
\end{tabular}




\section{TABLE VIII.}

HeLIUM. $-\mathrm{N}=2 \cdot 4 ; \mathrm{V}=14 \cdot 5$.

\begin{tabular}{|c|c|c|c|c|c|c|}
\hline $\mathrm{X}$. & 60 & 50 & 40 & 30 & 20 & 10 \\
\hline $\begin{array}{c}\alpha \text { found } \\
\text { experimentally. }\end{array}$ & 1.35 & $1 \cdot 20$ & 1.00 & .77 & $\cdot 40$ & $\cdot 12$ \\
$\mathrm{~N}_{\epsilon}-\frac{\mathrm{NV}}{\mathrm{X}}$ & 1.34 & $1 \cdot 20$ & 1.01 & .75 & .42 & $\cdot 074$ \\
\hline
\end{tabular}

The above tables include the results of the most accurate experiments, and are probably correct to within 2 or 3 per cent. The values of $a / p$ for the large values of $\mathrm{X} / p$ given in some of the papers are not very reliable, so they have not been included. The difficulty arises in these cases from the action of the positive ions. When the positive ions are not producing any appreciable effect the value of $a$ can always be determined accurately by taking the ratio of the currents at two different distances between the plates, even when the free path of the ion is a considerable fraction of the smaller distance, as it is only the change of distance which is involved in the calculation. With large forces and small pressures, however, it is necessary to take into consideration the action of the positive ions. In this case the expression for the ratios of the currents at different distances involves the absolute distance, and for accurate determinations it is desirable to use distances between the plates which would be large compared with the free paths. But there is a limit to the extent to which such experiments can be carried, for with large forces and small pressures sparking takes place when the distance 
exceeds a certain critical value. On this account the determinations of $a / p$ for values of $\mathrm{X} / p$ larger than those which are given in the above tables have not been obtained with a high degree of accuracy.

The tables show that in all cases the theory is in accordance with the experimental results over a wide range when the forces are large. For the smaller forces the values of $a$ are larger than those given by the expression $\mathrm{N} \epsilon-\frac{\mathrm{NV}}{\mathrm{X}}$. There are many causes which may contribute to this difference, but it is impossible to give any reliable explanation of it as so little is known as to what exactly takes place when an ion collides with a molecule.

It has been assumed that one effect of a collision is to reduce the velocity of an ion to a relatively small value, an assumption which seems reasonable when the velocities are so large that the ions tend to produce changes in the molecules by impact. In these cases a large proportion of the kinetic energy of the ion would be absorbed by the molecule, so that after a collision the ion would begin to move under the electric force with a small velocity. But when the velocities on impact are much below that which is required to ionize a molecule, there is no reason for supposing that a large proportion of the kinetic energy of the ion is lost on collision; it may thus acquire the critical velocity more frequently than is shown by the calculations for the smaller forces. It is also probable that there are other circumstances besides the velocity of the negative ion which determine what takes place on collision, and if so, that ions may be produced on some few occasions when the negative ion collides with a velocity less than that 
corresponding to the potential fall V. A theory worked out on these lines would make the expression for $a$ in terms of X very complicated, and would not add much to the information which is obtained by comparing the experiments with the simple formula involving one exponential term.

13. Molecular dimensions deduced from the mean free paths of negative ions.

The mean free paths of the negative ions in the different gases at 1 millimetre pressure are the reciprocals of the numbers N. As might be expected, the mean free path is longer in hydrogen than in other gases, and in general the sectional area of the molecule which is proportional to $\mathrm{N}$ is larger in the heavier gases. The most notable exception occurs with helium. Although the density of this gas is twice that of hydrogen, a negative ion makes twice as many $(5 \div 2 \cdot 4)$ collisions in travelling a given distance in hydrogen as it does in helium at the same pressure.

It is interesting to compare the mean free paths of the ions as obtained by this method with the mean free paths of molecules of gases obtained from the determinations of the viscosity. If a collision occurs between two molecules when their centres come within a distance $2 \sigma$ from each other, then a negative ion, being of small dimensions, would on this view collide with the molecule when it came within a distance $\sigma$ of the centre. It will, however, be seen that the ion makes fewer collisions than would occur under these circumstances, or, in other words, that the ion must come within a distance $\mathrm{R}$ from 
the centre of the molecule in order that ionization may result from the collision, the distance $R$ being less than $\sigma$. For if $m$ is the number of molecules in a cubic centimetre of a gas at 760 millimetres pressure and $15^{\circ} \mathrm{C}$., then $\pi \mathrm{R}^{2} m=\mathrm{N} \times 760$, and the quantity $\pi \sigma^{2} m$ may be deduced from the coefficients of viscosity. The values of $R \times 10^{8}$ and $\sigma \times 10^{8}$ in centimetres for the different gases are given in the following table, the number $m$ being taken as $3 \times 10^{19}$ which is the value deduced from the recent investigations by Professor Perrin. ${ }^{1}$ The values of $\sigma$ have been deduced for the same value of $m$ from the expression for the viscosity given by Professor Jeans. ${ }^{2}$

TABLE IX.

\begin{tabular}{|c|c|c|c|c|c|c|c|c|}
\hline & Air & $\mathrm{N}_{2}$ & $\mathrm{H}_{2}$ & $\mathrm{CO}_{2}$ & $\mathrm{HCl}$ & $\mathrm{H}_{2} \mathrm{O}$ & A & $\mathrm{He}$ \\
\hline $\mathrm{N}$ & $14 \cdot 6$ & $12 \cdot 4$ & $5 \cdot 0$ & $20 \cdot 0$ & $22 \cdot 2$ & $12 \cdot 9$ & $13 \cdot 6$ & $2 \cdot 4$ \\
\hline $\mathrm{R} \times 10^{8}$ & $\begin{array}{l}1.08 \\
1.64\end{array}$ & $\begin{array}{l}1.00 \\
1.67\end{array}$ & $\begin{array}{r}\cdot 63 \\
\cdot 18\end{array}$ & \begin{tabular}{|l|}
$1 \cdot 26$ \\
0.00
\end{tabular} & $1 \cdot 33$ & $\begin{array}{l}1.02 \\
1.09\end{array}$ & $1 \cdot 04$ & $\cdot 44$ \\
\hline & & & & & & & & \\
\hline
\end{tabular}

14. Application of the theory to Stoletow's experiments.

The experiments which have been described were arranged expressly for the purpose of obtaining results from which the values of $a$ could be easily obtained, as no reliable investigations had previously been made

1 Jean Perrin, Comptes Rendus, Octobre 5, 1908.

2 Jeans, "Dynamical Theory of Gases," p. 250. The values of $10^{8} \sigma$ given in this treatise on p. 251 correspond to $m=4 \times 10^{18}$. 
from which these values could be deduced. Several experimental investigations, however, have been made by other physicists of the conductivity produced by external agencies in gases at low pressures. Among the earliest were those by Stoletow, ${ }^{1}$ who showed that there are large variations in the currents between parallel plates due to changes in the force and pressure when ultra-violet light acts on the negative electrode.

Most of the effects which were observed may obviously be attributed to the development of large currents by the collisions of the ions with the molecules of the gas, and a detailed examination of all these experiments is unnecessary. One result, however, obtained by Stoletow, may be mentioned, as it requires some consideration to see how it can be explained by the theory.

It was found that when the distance and potential fall between the electrodes is constant, the current increases as the pressure of the gas is diminished, that at a certain pressure $P$ the current attains a maximum value, and that when the pressure is reduced below the value $\mathrm{P}$ the current diminishes. The pressure $P$, at which the current attains the maximum value, is proportional to the force $\mathrm{X}$ and independent of the distance between the plates.

The following table is given by Stoletow for air, ${ }^{2} \mathrm{E}$ being the electromotive force between the two plates, the unit being the electromotive force of a Clark cell, $l$ the distance between the plates in millimetres, and $\mathrm{P}$ the pressure,

1 Stoletow, Comptes Rendus, t. crii., p. 91, 1888; Journal de Physique, Série ii., t. ix., 1890.

2 Stoletow, Journal de Physique, loc. cit. 
in millimetres of mercury, for which the current is a maximum.

TABLE X.

\begin{tabular}{|c|c|c|c|}
\hline $\mathrm{E}$ & $l$ & $\mathrm{P}$ & $\mathrm{P} l$ \\
& & & $\mathrm{E} \times 10^{4}$ \\
\hline & & & \\
\hline $16 \tilde{5}$ & $\cdot 2 \tilde{5}$ & $25 \cdot 3$ & 383 \\
$16 \tilde{5}$ & $\cdot 47$ & $13 \cdot 5$ & 384 \\
$6 \tilde{5}$ & $\cdot 47$ & $\cdot \bar{\cdot} \cdot 3$ & 383 \\
100 & $\cdot 83$ & $4 \cdot 7$ & 389 \\
$6 \tilde{5}$ & $\cdot 83$ & $3 \cdot 0$ & 383 \\
60 & $\cdot 83$ & $2 \cdot 8$ & 386 \\
$6 \tilde{5}$ & $1 \cdot 91$ & $1 \cdot 3$ & 382 \\
$6 \tilde{5}$ & $3 \cdot 71$ & $\cdot 67$ & 382 \\
40 & $3 \cdot 60$ & $\cdot 43$ & 387 \\
\hline
\end{tabular}

The agreement between the figures in the last column shows that $\mathrm{P}$ is proportional to the force $\mathrm{E} / l$.

When $\mathrm{E}$ is expressed in volts and $l$ in centimetres, the value of the pressure for which the current is a maximum is $\mathrm{P}=\frac{\mathrm{X}}{372}$. In these experiments the potentials and the distances between the plates were small, so that there can be no appreciable effect due to ionization by the action of positive ions. There may be a small variation of the number of ions set free from the plate due to the variation in the pressure, but the principal variations must be due to the ionization produced by the motion of the negative ions. The pressure for which the current is a maximum should therefore be practically the same as that at which $a$ becomes a maximum. This pressure can be deduced by differentiating the formula, $a=p f\left(\frac{\mathrm{X}}{p}\right)$, 
with respect to $p$ and equating $\frac{d a}{d p}$ to zero. The following condition for a maximum value of $a$ is thus obtained :

$$
f\left(\frac{\mathrm{X}}{p}\right)-\frac{\mathrm{X}}{p} f^{\prime}\left(\frac{\mathrm{X}}{p}\right)=0 .{ }^{1}
$$

It is obvious that $\mathrm{P}$ is proportional to $\mathrm{X}$ since the equation involves only the ratio $\mathrm{X} / p$. In order to find the value of $\mathrm{X} / \mathrm{P}$ which satisfies this equation it is not necessary to know the form of the function $f$ as the required ratio may be found for each gas from the curves (figures 6 and 7). The $x$ and $y$ co-ordinates of any point on one of the curves being $\mathrm{X} / p$ and $a / p$ respectively, it follows from the above condition that $y=x \frac{d y}{d x}$ at the point corresponding to the maximum value of $a$. The equation $\frac{d y}{d x}=\frac{y}{x}$ shows that the tangent to the curve at the point $(x, y)$ must pass through the origin, so that $x$ and $y$ are obtained by drawing a tangent to the curve from the origin. In the curve for air the point of contact of the tangent is near the point where $\mathrm{X} / p$ has the value 370 , and this value is in good agreement with the result obtained by Stoletow. It is not easy, however, to judge the exact point at which the tangent touches the curve, so that the following method is perhaps more satisfactory.

1 See paper by the author, Philosophical Magazine, February, 1901; also J. J. Thomson, "Conduction of Electricity through Gases," 1903 edition, pp. 233, 342, where the same result is given in a different notation. 
15. Determination of the pressure corresponding to the maximum value of $a$ for a given force.

Since the values of $a / p$ determined experimentally agree very closely with those given by the formula $\frac{a}{p}=\mathrm{N} \epsilon \frac{\mathrm{NV} p}{\mathrm{X}}$ for the larger values of $\mathrm{X} / p$, the pressure for which $a$ is a maximum when $\mathrm{X}$ is constant may be obtained by equating to zero the differential coefficient of $p \epsilon^{-\frac{\mathrm{NV} p}{\mathrm{X}}}$ with respect to $p$. The equation in $\mathrm{X} / p$ thus obtained is $1-\frac{\mathrm{NV} p}{\mathrm{X}}=0$.

Thus for any gas the pressure $\mathrm{P}$, which gives the greatest conductivity with a force $X$, is $P=\frac{X}{\overline{N V}}$, and the maximum value of $a$ is $\mathrm{NP}^{-1}=\frac{\mathrm{X}}{\mathrm{V} \epsilon}$.

The factor by which the original number of ions is multiplied owing to the effects of collisions depends on al or $\frac{\mathrm{X} l}{\mathrm{~V} \epsilon}=\frac{v}{\mathrm{~V} \epsilon}$, where $v$ is the potential difference between the plates. Thus in the case in which $n_{0}$ ions are set free from the negative electrode the number that arrive at the positive electrode has a maximum value $n_{0} \epsilon^{\frac{v}{v_{\epsilon}}}$, independent of the distance $l$ between the plates when a given potential difference $v$ is established between them. In order that this equation may hold accurately it is necessary that the negative ions should have the mean velocity corresponding to the force $\mathrm{X}$ along the whole distance $l$. This condition will not be established until the ions have travelled a short distance from the 
negative electrode; beyond this it holds, so that the factor by which the current is multiplied by increasing the distance from $l_{1}$ to $l_{2}$ is given accurately by the term $\epsilon \frac{\mathrm{x}\left(l_{2}-l_{1}\right)}{\mathrm{V}_{e}}$, and this result is confirmed by experiment. The potential difference between the plates when the first distance $l_{1}$ is used should not be less than $\mathrm{V}$, or $l_{1}$ should be greater than $\mathrm{V} / \mathrm{X}$, and for the larger distances the potential $l \mathrm{X}$ should not approach the sparking potentials, otherwise the effects produced by positive ions would be appreciable.

Table XI. gives the values of $\mathrm{V}$ and $\mathrm{NV}$ for the different gases.

TABLE XI.

\begin{tabular}{|c|c|c|c|c|c|c|c|c|}
\hline & AIR & $\mathrm{N}_{2}$ & $\mathrm{H}_{2}$ & $\mathrm{CO}_{2}$ & HCl. & $\mathrm{H}_{2} \mathrm{O}$ & A & $\mathrm{He}$ \\
\hline $\mathrm{V}$ & 25 & $27 \cdot 6$ & 26 & $23 \cdot 3$ & 16.5 & $22 \cdot 4$ & $17 \cdot 3$ & $14 \cdot 5$ \\
\hline NV & 365 & 342 & 130 & 466 & 366 & 289 & 235 & $34 \cdot 8$ \\
\hline
\end{tabular}

The greatest conductivity is obtained with a force $\mathrm{X}$ when the pressure in millimetres $\mathrm{P}=\frac{\mathrm{X}}{\mathrm{NV}}, \mathrm{X}$ being expressed in volts per centimetre. For air this theory gives $\mathrm{P}=\frac{\mathrm{X}}{365}$ which is in good agreement with Stoletow's result $\mathrm{P}=\frac{\mathrm{X}}{372}$.

The maximum value of $a$ is $\frac{\mathrm{X}}{\epsilon \mathrm{V}}$, or $a l=\frac{v}{\epsilon \overline{\mathrm{V}}}, v$, the potential difference between the plates, being expressed in volts, and $l$ in centimetres. 
It thus appears that with a given potential $v$ the best conductivity is obtained with those gases for which $\mathrm{V}$ is small, as with helium, hydrochloric acid, and argon. The second of these gases would give results in accordance with the formulæ over large ranges of potentials, since positive ions do not produce appreciable effects in this gas until fairly large potentials are attained.

\section{Comparison of the velocities of ions and molecules.}

It is interesting to see how the velocity acquired by an ion moving freely under an electric force compares with the velocity of agitation when its motion is in equilibrium with the surrounding molecules of a gas at normal temperature, $15^{\circ} \mathrm{C}$. This point has been examined by Professor Schuster in one of his Bakerian ${ }^{1}$ lectures, and the following is essentially the method adopted.

If $v$ is the velocity acquired by an ion of mass $\mathrm{M}$ in travelling freely along a path over which the potential difference is $\mathrm{V}$, then $\frac{1}{2} \mathrm{M} v^{2}=\frac{e \mathrm{~V}}{300}$, the charge $e$ being expressed in electrostatic units, and $\mathrm{V}$ in volts. The velocity of agitation $u$ is given by the equation $\frac{1}{3} \mathrm{M} m u^{2}=10^{6}$ where $m$ is the number of molecules in a gas at normal pressure and temperature. Hence

$$
\frac{v^{2}}{u^{2}}=27 \mathrm{~V}, q \cdot p \text {. }
$$

me being $1.23 \times 10^{10}$.

Hence in order to generate ions by collision it is necessary that the velocity of the negative ion should be

1 Proceedings of the Royal Society, Vol. xlvii., 1890. 
much greater than the velocity of agitation that it would have in a gas at $15^{\circ} \mathrm{C}$.

The velocity acquired by a negative ion under the action of the potential difference $V$ may be also compared with the velocity of the $\beta$ particles emitted by radioactive bodies, or with that of the cathode rays. In the former case some of the particles travel with a velocity of $2.85 \times 10^{10}$ centimetres per second, and in the latter case the velocity is much smaller, and depends on the circumstances under which they are generated. The ratio of the charge $e$ to the mass $\mu$ of the particle has been found by Simon ${ }^{1}$ to be $1.86 \times 10^{7}$ for cathode particles travelling with an average speed of $7 \times 10^{9}$ centimetres per second, $e$ being expressed in electro-magnetic units.

The potential required to impart a velocity of $7 \times 10^{9}$ centimetres per second to a particle for which the ratio $\frac{e}{\mu}$ has this value is 13,000 volts, which is large compared with the voltages required to generate ions by collisions. Taking the latter to be of the order of 20 volts and the ratio $\frac{e}{\mu}$ to have the above value for negative ions generated by collisions in gases, the velocity they require to generate others by collisions is of the order of $2.7 \times 10^{8}$ centimetres per second.

1 Simon, Wied. Annal., p. 589, 1899. 


\section{CHAPTER II}

IONIZATION BY POSITIVE IONS

17. Conductivity between parallel plates when positive and negative ions generate others by collisions.

The effects produced by the motion of positive ions may be deduced from determinations of the currents that pass between parallel plates while ultra-violet light is falling on the negative electrode, if the forces and the distances between the plates are sufficiently large.

It has already been shown that when $\mathrm{X} / p$ and $l$ are small, and $l$ is varied whilst $\mathrm{X}$ and $p$ are kept constant, the number of negative ions reaching the positive electrode is $n_{0} \epsilon^{a l}$, where $a$ is a constant depending on $\mathrm{X}$ and $p$. For large values of $\mathrm{X} / p$ and $l$ the number of negative ions reaching the positive electrode is greater than $n_{0} \epsilon^{\alpha l}$, showing that some other form of ionization has come into play. This stage is attained even when the potential between the plates is much less than that required to produce a spark. It will be seen from the following investigations that all the features of the new process of ionization can be explained on the supposition that it arises from the action of the positive ions.

Further, it is obvious that if both positive and negative ions produce others in sufficient numbers, a current would be obtained which would last after the supply 
from the negative electrode was cut off, and a continuous discharge would ensue. The investigations show how the potential required to produce a continuous discharge may be found on the assumption that the whole ionization is produced by collisions of positive and negative ions in a gas, and, as will be seen, there is a very accurate agreement over a large range of pressures between the potentials thus calculated and the sparking potentials determined experimentally.

In making an investigation of the currents which would be produced between parallel plates when both positive and negative ions generate others by collisions, the results of the experiments may be anticipated, and it may be assumed that the positive and negative ions produced by the impact of a positive ion with a neutral molecule are identical with the positive and negative ions produced by the impact of a negative ion. In applying the theory, therefore, it is necessary only to consider one kind of positive ion and one kind of negative ion in each gas, but the positive ions, unlike the negative ions, are most probably different in different gases.

If a number $n_{0}$ of negative ions start from the negative plate and move through a distance $l$ from the positive plate they will generate others in the gas, and a number $n_{0} \epsilon^{a l}$ will reach the positive electrode. Thus $n_{0}\left(\epsilon^{a l}-1\right)$ positive ions are produced in the gas and move in the opposite direction. When these also ionize the gas the total number $n$ of negative ions that reach the positive electrode exceeds $n_{0} \epsilon$.

Of the number $n-n_{0}$ of each kind generated in the gas, let $r$ be produced in the layer of gas between the negative plate and a parallel plane at a distance $x$, and 
let $r^{\prime}$ be produced in the layer between this plane and the positive electrode.

$$
\text { Then } n=n_{0}+r+r^{\prime} \text {. }
$$

Let $a$ be the number of ions produced by a negative ion in moving through one centimetre of the gas.

Let $\beta$ be the corresponding number for a positive ion.

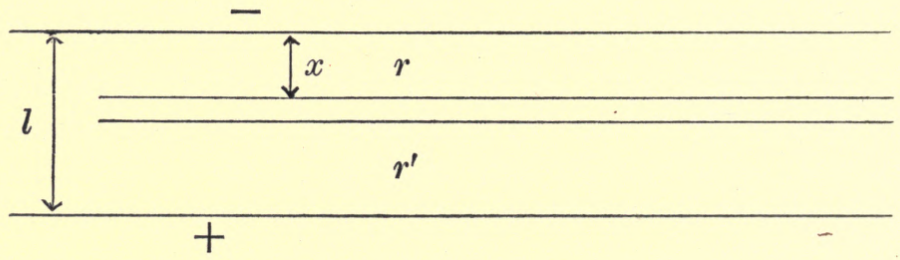

The number of ions $d r$ generated between the two planes at distances $x$ and $x+d x$ is given by the expression $d r=a\left(n_{0}+r\right) d x+\beta r^{\prime} d x$, since the number of negative ions travelling through the length $d x$ is $n_{0}+r$, and these produce $a\left(n_{0}+r\right) d x$, and the number of positive ions passing through the same element of length $d x$ is $r^{\prime}$, and they produce $\beta r^{\prime} d x$. Substituting $n-n_{0}-r$ for $r^{\prime}$ the equation for $r$ becomes

$$
\frac{d r}{d x}=(\alpha-\beta)\left(n_{0}+r\right)+\beta n,
$$

which on integration gives

$$
n_{0}+r=-\frac{\beta n}{a-\beta}+c \epsilon^{(a-\beta) x} .
$$

The constant $c$ is determined by the condition $r=0$ when $x=0$.

Hence the value of $r$ is given by the equation

$$
n_{0}+r=-\frac{\beta n}{a-\beta}+\left(n_{0}+\frac{\beta n}{a-\beta}\right) \epsilon^{(a-\beta) x},
$$


and the value of $n$ in terms of the other constants is obtained by substituting for $r$ its value $n-n_{0}$ when $x=l$, $l$ being the distance between the plates. Thus

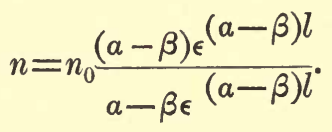

This gives the number of negative ions $n$ arriving at the positive electrode when $n_{0}$ negative ions start from the negative electrode, and both positive and negative ions generate others by collisions with molecules of the gas.

It will be noticed that when the distance between the plates $l$ has a certain value $\mathrm{S}$ given by the equation

$$
\alpha-\beta \epsilon^{(a-\beta) \mathrm{S}}=0, \text { or } \mathrm{S}=\frac{1}{a-\beta} \log \left(\frac{a}{\beta}\right),
$$

the denominator of the fraction in the expression for $n$ becomes zero, so that $n$ becomes infinite. This shows that a current would continue to flow indefinitely after the supply of the negative ions $n_{0}$ from the surface of the negative electrode ceases. The importance of this conclusion in connection with sparking potentials will be considered later. ${ }^{1}$

For distances between the plates shorter than $\mathrm{S}$ the denominator of the fraction expressing $n$ is positive, and the current becomes zero after the light ceases to act on the electrode. The values of $n$ are then finite, but greater than the number of ions $n_{0} \epsilon^{a l}$ which would reach the positive electrode if the negative ions alone produced others by collisions.

1 See section 23. 


\section{Agreement between experiments and theory.}

To illustrate the theory the experiments ${ }^{1}$ may be quoted in which the currents between two parallel plates in air at one millimetre pressure were determined, the electric force being 350 volts per centimetre in each case. The distance between the plates $l$ is given in centimetres in the first line of Table XII.; the second line gives the charge $q$ acquired by one of the plates while the light was acting on the negative electrode for a fixed time; the third and fourth lines give the values

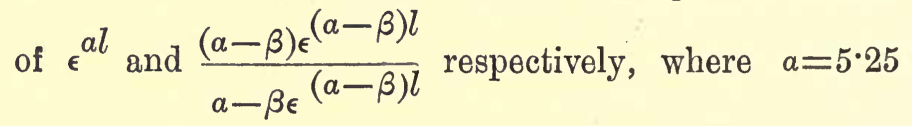
and $\beta=\cdot 0141$.

The values of $\epsilon^{a l}$ agree only with the experiments at the shorter distances, but the numbers in the fourth line show that the positive ions (although $\beta$ is small compared with $a$ ) produce an appreciable effect when the plates are about 6 millimetres apart and have a very large effect for larger distances.

TABLE XII.

\begin{tabular}{|c|c|c|c|c|c|c|c|}
\hline$l$ & 0 & $\cdot 2$ & $\cdot 4$ & $\cdot 6$ & $\cdot 8$ & $1 \cdot 0$ & $1 \cdot 1$ \\
$q$ & - & $2 \cdot 86$ & $8 \cdot 3$ & $24 \cdot 2$ & 81 & 373 & 2250 \\
$\epsilon^{\alpha l}$ & 1 & $2 \cdot 86$ & $8 \cdot 2$ & $23 \cdot 4$ & $66 \cdot 5$ & 190 & 322 \\
$\frac{(\alpha-\beta) \epsilon^{(\alpha-\beta) l}}{\alpha-\beta \epsilon}{ }^{(a-\beta) l}$ & 1 & $2 \cdot 87$ & $8 \cdot 3$ & $24 \cdot 8$ & 80 & 380 & 2150 \\
\hline
\end{tabular}

1 See papers in Electrician, April 3, 1903, and Philosophical Magazine, November, 1903, on "The Genesis of Ions by the Motion of Positive Ions in a Gas." 
The currents are given in arbitrary units, and the values of $\alpha$ and $\beta$ may be found from the ratios of the currents at three distances. The agreement between the expression for $n$ involving $a$ and $\beta$ and the currents over several distances shows that the experiments are accurately explained by the theory.

The above example of currents developed in air, in which $p=1, \mathrm{X}=350, a=5 \cdot 25$, and $\beta=\cdot 0141$, may be compared with the results of an experiment in which $p=2, \mathrm{X}=700$, since according to the theory $\alpha$ and $\beta$ should in this case have twice the above values, and the current corresponding to any distance should be the same as that at double the distance in the previous experiment.

Table XIII. gives the values of the currents $q$ in this case.

TABLE XIII.

\begin{tabular}{|c|c|c|c|c|c|}
\hline$l$ & $\cdot 1$ & $\cdot 2$ & $\cdot 3$ & $\cdot 4$ & $\cdot 5$ \\
$q$ & $2 \cdot 9$ & $8 \cdot 3$ & $23 \cdot 8$ & 80 & 374 \\
$\frac{(\alpha-\beta) \epsilon(\alpha-\beta) l}{\alpha-\beta \epsilon}(\alpha-\beta) l$ & $2 \cdot 87$ & 8.3 & $24 \cdot 6$ & 80 & 380 \\
\hline
\end{tabular}

The third line gives the calculated values when $a=10.5$ and $\beta=0282$. The currents at $1,2,3,4$, and 5 millimetres are the same as those in the previous experiment when the distances were double these values.

For comparison with other gases the following examples may be taken from among the experiments which have been made with hydrogen. 
TABLE XIV.

Hrdrogen. $-p=2$ mms.; $\mathrm{X}=262$ volts per cm.; $a=3 \cdot 7 ; \beta=\cdot 041$.

\begin{tabular}{c|r|r|r|r}
$l$ & 4 & $\cdot 6$ & $\cdot 8$ & $1 \cdot 0$ \\
$q$ & $4 \cdot 6$ & $10 \cdot 0$ & $22 \cdot 7$ & 65 \\
\hline$(\alpha-\beta) \epsilon^{(\alpha-\beta) l}(\alpha-\beta) l$ & 4.55 & 9.9 & $22 \cdot 7$ & 67 \\
\hline$\alpha-\beta \epsilon$
\end{tabular}

TABLE XV.

Hydrogen. $-p=4$ mms.; $\mathrm{X}=525$ volts per cm.; $a=7 \cdot 4 ; \beta=\cdot 082$.

\begin{tabular}{|c|c|c|c|c|}
\hline$l$ & $\cdot 2$ & $\cdot 3$ & $\cdot 4$ & $\cdot 5$ \\
$q$ & $4 \cdot 6$ & $9 \cdot 9$ & $22 \cdot 3$ & 66 \\
$\frac{(\alpha-\beta) \epsilon^{(\alpha-\beta) l}}{\alpha-\beta \epsilon}(\alpha-\beta) l$ & $4 \cdot 55$ & 9.9 & $22 \cdot 7$ & 67 \\
\hline
\end{tabular}

Several experiments of this kind have been made with a number of gases, and they all show that when $\mathrm{X}$ and $p$ are multiplied by a factor the values of both $a$ and $\beta$ are multiplied by the same factor. This agrees with the theory which requires that the connection between $\beta$ and the variables $\mathrm{X}$ and $p$ should be of the same form as the relation $\frac{a}{p}=f\left(\frac{\mathrm{X}}{p}\right)$, so that $\frac{\beta}{p}=\phi\left(\frac{\mathrm{X}}{p}\right)$. The values of $\beta$ may therefore be obtained from a curve whose ordinates are $\beta / p$ and $\mathrm{X} / p$.

A point to be noticed is that with a given potential 
and a given quantity of gas between the plates the current is independent of the distance $l$. This follows immediately from elementary considerations, for when the number of molecules between the plates is constant an ion makes a fixed number of collisions, and the fall of potential along each path depends only on the potential difference between the plates. Under these circumstances the total number of ions generated by positive and negative ions must be independent of the distance between the plates.

19. Curves representing $\beta / p$ as a function of $\mathrm{X} / p$; comparison of effects produced by positive and negative ions.

The values of $\beta / p$ in terms of $\mathrm{X} / p$ for the different gases are shown by the curves figure 9 . The numbers obtained for $\beta$ in argon and nitrogen are practically the same, so that a single curve is given for the values of $\beta / p$ for these two gases. ${ }^{1}$

It will be observed that for the same force and pressure $\beta$ is much less than $a$ in any gas. It can be

1 The values of $\beta / p$ for helium are probably not as accurate as those for other gases. They have been deduced from the values of $\alpha$ and a few determinations of the sparking potential obtained for pure helium (E. W. B. Gill and F. B. Pidduck, Philosophical Magazine, August, 1908). When the sparking potential $\mathrm{V}$ is known for a given pressure $p$ and distance $\mathrm{S}$ between the plates, then $\frac{\mathrm{V}}{p \mathrm{~S}}=\frac{\mathrm{X}}{p}$, and the corresponding value of $\alpha$ can be found from the curve, giving $\alpha / p$ in terms of $\mathbf{X} / p$. The value of $\beta$ may then be deduced from the equation $\alpha-\beta \epsilon^{(\alpha-\beta) \mathrm{S}}=0$. The presence of impurities in helium gives rise to comparatively large errors in the values of $\alpha$ and $\beta$, and it is intended to make further experiments in which particular care will be taken to maintain the helium free from impurities. 
shown from the results that have been obtained that positive ions are much less efficient than negative ions in producing new ions by collision even when they have the same kinetic energy. For, assuming that the positive ions are large compared with negative ions and of the same order of magnitude as the molecules of the

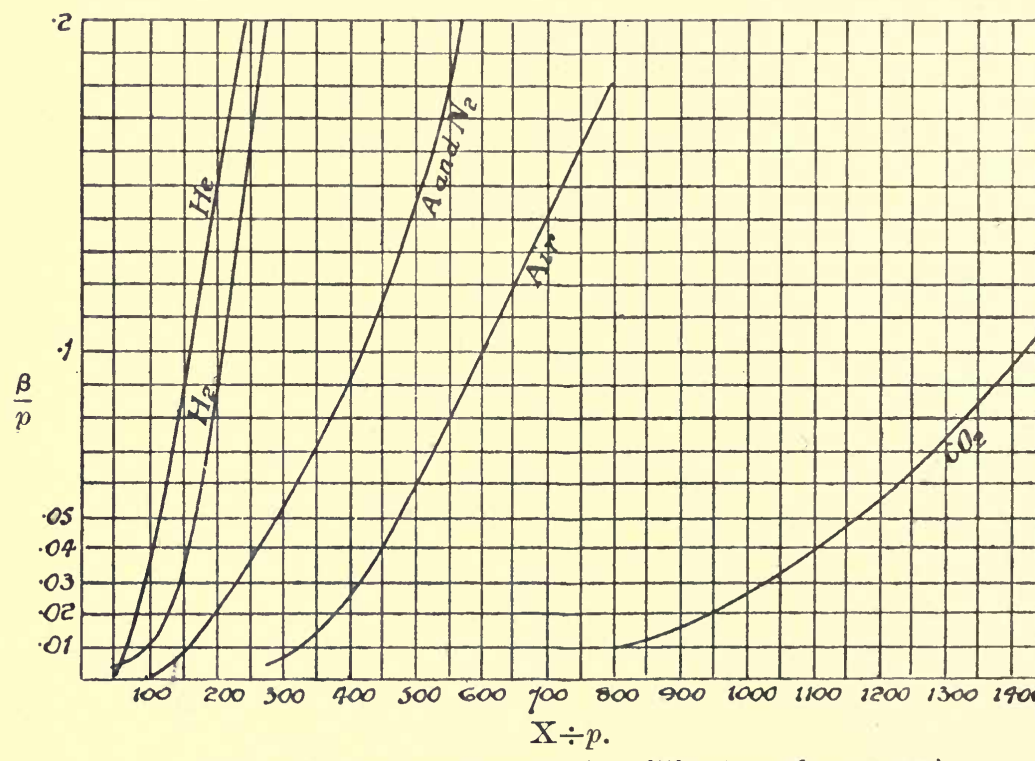

( $\mathrm{X}$ in volts per centimetre, $p$ in millimetres of mercury.)

Figure 9.

gas from which they are derived, each positive ion would make four times as many collisions with molecules as a negative ion in travelling the same distance in the gas. The free paths of a negative ion in a gas at pressure $p$ will therefore be the same as the free paths of a positive ion in a gas at pressure $p / 4$, and if the same electric force 
$\mathrm{X}$ be acting in each case the energy acquired between the collisions will also be the same. The relative ionizing powers may therefore be found by comparing the value of $a$ in a gas at a certain pressure with the value of $\beta$ in the gas at a quarter of the pressure when a constant force $X$ is acting. Take as an example the case of hydrogen. With the force $\mathrm{X}=50$ volts per centimetre and pressure 1 millimetre $a=\cdot 35$. The curves giving $\beta$ show that $\frac{\beta}{p}=\cdot 090$ when $\frac{\mathrm{X}}{p}=200$, so that $\beta=\cdot 022$ when $\mathrm{X}=50$ and $p=\cdot 25$. Hence when positive and negative ions collide an equal number of times with molecules and acquire equal kinetic energies along their free paths the negative ion produces sixteen times as many ions as the positive ion. The ratio of the activities of the positive and negative ions as estimated in this way varies with the force $X$. Under a force of 75 volts per centimetre $a=\cdot 9$ when $p=1$, and $\beta=\cdot 065$ when $p=\cdot 25$, so that the effect of the negative ion is fourteen times that of the positive ion.

The rate of increase of the ionizing power of positive ions is therefore greater than that of negative ions, and for very large forces $\beta / p$ would theoretically be four times as great as the maximum value of $a / p$. But it is impossible to test the accuracy of the theory on this point, since the smaller values of $\beta$ do not supply sufficient data for the purpose, and the larger values cannot be obtained by the experimental method that has been used, as sparking takes place before $\beta / p$ rises above a small fraction. The above figures nevertheless show that for small values of the kinetic energy the negative ions are much more efficient than positive ions in producing new ions by collision. 
If this difference between the positive and negative ions is due to the relatively large mass of the former, it is to be expected that the difference would be more marked in the heavier gases, assuming that the mass of the positive ion is of the same order as that of a molecule of the gas. It is interesting, therefore, to make similar calculations for other gases and to compare them with the results obtained for hydrogen. For although these comparisons give no accurate information as to the ratio of the masses of the positive ions in different gases, the calculations afford some reason for believing that the positive ions in other gases are greater than those in hydrogen.

A matter of importance that arises in making comparisons of the relative activity of positive and negative ions in different gases is to decide what force to select in each case, as the relative activity varies with the force. The principle which is here adopted is to take in each case such a force as will give a value of $a$ in the gas at a millimetre pressure that is a definite fraction of $\mathrm{N}$, the maximum value of $a$. Thus in the examples that have already been given for hydrogen at

$$
\begin{aligned}
50 \text { volts per centimetre } a & =\cdot 35=\cdot 07 \mathrm{~N}, \\
75, \quad a & =\cdot 9=\cdot 18 \mathrm{~N},
\end{aligned}
$$

$\mathrm{N}$ being equal to five for hydrogen.

Taking the forces that give $a=16 \mathrm{~N}$ in each case, the following numbers may be obtained from the experimental results. For air $\mathrm{N}=14^{\circ} 6$, and the force which corresponds to $a=14.6 \times \cdot 16=2.33$ is 190 volts per centimetre when $p=1$. The value of $\beta$ when $\mathrm{X}=190$ and $p=.25$ is obtained from the point on the curve corresponding to $\frac{\mathrm{X}}{p}=760$. This gives $\frac{\beta}{p}=\cdot 164$, so 
that $\beta=\cdot 041$. These values of $a$ and $\beta$ are in the ratio of $57: 1$. Again, for argon $\mathrm{N}=13 \cdot 6$, and

$$
\begin{aligned}
& a=2 \cdot 17 \text { when } X=105 \text { and } p=1, \\
& \beta=\cdot 025 \text { when } X=105 \text { and } p=\cdot 25,
\end{aligned}
$$

giving the ratio $a: \beta=86: 1$.

For carbon dioxide $\mathrm{N}=20$ and

$$
\begin{aligned}
& a=3 \cdot 2 \text { when } \mathrm{X}=215 \text { and } p=1, \\
& \beta=\cdot 003 \text { when } \mathrm{X}=215 \text { and } p=\cdot 25
\end{aligned}
$$

giving the ratio $a: \beta=1070: 1$.

It thus appears that the greater the molecular weight of the gas the greater the difference between positive and negative ions as regards their efficiency in producing new ions by collisions.

In the case of pure helium the range of forces for which observations of $a$ and $\beta$ have been made is very small, and it would not be possible to deduce numbers from them similar to those found for other gases at the point at which $a=\cdot 16 \mathrm{~N}$. The values of $a$ and $\beta$ that have been found for an impure specimen of the gas containing about 98 per cent. of helium may be used. They give the following results :

$$
\begin{array}{ll}
a=\cdot 5, & \mathrm{X}=25, p=1, \\
\beta=\cdot 0095, & \mathrm{X}=25, p=\cdot 25,
\end{array}
$$

which gives $a=52 \times \beta$.

This result shows that the positive ions in helium are less efficient than those in hydrogen, but it is remarkable that the ratio of $a$ to $\beta$ should be nearly the same as for air.

When comparisons of the relative efficiency of positive and negative ions in pure helium and in air are made, using smaller forces which correspond to values of $a$

I.G. 
equal to $\cdot 1 \times \mathrm{N}$ in each case, a similar result is obtained $a$ being $70 \beta$ for both gases.

Again in nitrogen the range of values of $\beta$ does not admit of comparisons being made at the point $\alpha=\cdot 16 \mathrm{~N}$, but for $a=\cdot 1 \times \mathrm{N}$ the corresponding value of $\beta$ gives $a=26 \beta$.

The relative ionizing powers of negative and positive ions in different gases thus obtained are-for hydrogen $15: 1$, air $57: 1$, argon $86: 1$, carbon dioxide $1070: 1$, and helium $52: 1$. The first four numbers may be said to support the assumption that the positive ions are of the same dimensions as the molecules, since the positive ions differ most from the negative for the gases with large molecular weights. 


\section{CHAPTER III}

SPARKING POTENTIAL IN A UNIFORM ELECTRIC FIELD

20. Description of phenomena accompanying discharges; sparking potential.

The various phenomena relating to the discharge of electricity through gases at low pressures have been investigated experimentally by many physicists, and the principal facts in connection with them have been well established. With regard to the sparking potential, it will be seen that the researches which have been carried out on the effects of collisions, suggest an obvious method ${ }^{1}$ of obtaining a simple theory of the sparking potentials for parallel plates in a gas at different pressures and also a consistent explanation of the large difference that exists between the sparking potential and the potential required to maintain the discharge at the higher pressures.

The principal features of the phenomena which are to be considered may be briefly stated as follows:-When a potential difference is established between two electrodes in a gas, the gas behaves as an insulator unless the potential exceeds a certain definite value, which is called the sparking potential. The sparking potential is very sharply defined, and it has been found that the

1 See papers by the author in Electrician, April 3, 1903, and Philosophical Magazine, November, 1903, and March, 1905. 
gas insulates well for a potential which is a few volts less than that required to initiate a discharge. When the applied potential is equal to or greater than the sparking potential, the insulation breaks down, and a comparatively large current, accompanied by a glow, passes through the gas. For a fixed distance between the electrodes the sparking potential changes with the pressure of the gas. At high pressures the potential is large; it diminishes with the pressure and reaches a minimum for a certain critical value of the pressure.

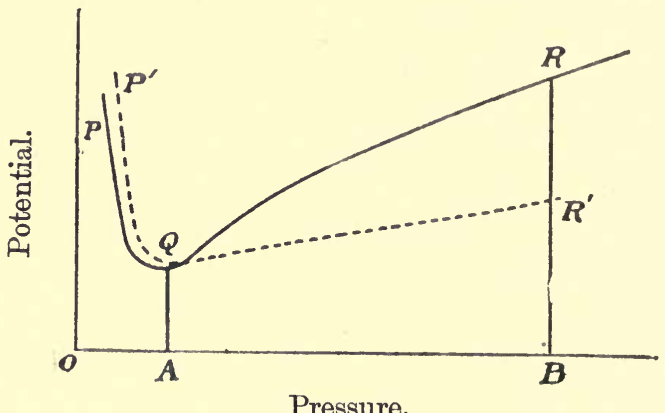

Pressure.

Figure 10.

When further reductions are made in the pressure the sparking potential rises rapidly, and attains very high values for the lower pressures.

The variations in the sparking potential may be illustrated by the curve, P Q R, figure 10 , O A being the critical pressure and $\mathrm{Q} A$ the minimum sparking potential.

When parallel plates are used as electrodes the sparking potential depends only on the quantity of gas between the electrodes, which is measured by the product of the pressure $p$ and distance $\mathrm{S}$ between the electrodes. This 
is known as Paschen's ${ }^{1}$ law, and it has been found by Carr ${ }^{2}$ to hold for large ranges of low pressures in the neighbourhood of the critical pressure.

All the experimental results at different distances and pressures may therefore be represented by a single curve, whose ordinates are the sparking potential and the product of the pressure and distance between the plates. In particular, it will be noticed that the critical pressure corresponding to the minimum sparking potential is inversely proportional to the distance between the plates.

\section{Potential required to maintain a discharge.}

When a spark discharge takes place between parallel plate electrodes the gas immediately becomes a conductor, and allows a comparatively large current to pass through. When the pressure is greater than the critical pressure the potential required to maintain the current is very much less than the sparking potential, and the potential difference between the electrodes diminishes as the current increases. When the pressure is less than the critical pressure a small current is produced when the sparking potential is applied, and in order to increase the current it is necessary to increase the potential difference between the electrodes. ${ }^{3}$

The potential required to maintain a current may be represented by the dotted line $P^{\prime} Q R^{\prime}$. At a point $B$, where the pressure is greater than the critical pressure, the gas insulates until the potential is equal to $R B$. If this potential be applied by a battery of cells through a resistance, immediately the spark occurs a large current

${ }^{1}$ Paschen, Wied. Ann., xxxvii., 1889.

${ }^{2}$ W. R. Carr, Philosophical Transactions A, Vol. cci., pp. 403433, 1903.

8 J. A. Brown, Philosophical Magazine, September, 1906. 
passes through the gas, and the potential difference at the electrodes falls to $R^{\prime} B$.

The following example of an experiment ${ }^{1}$ made with air at 4.31 millimetres pressure between parallel plates at 8 millimetres apart illustrates the phenomena. The gas insulated when a potential difference of 601 volts was established between the electrodes, but a spark took place when the potential was raised to 603 volts, and a current of 0052 ampère was established, which was maintained by a potential fall of 350 volts between the electrodes, the remainder of the 603 volts being taken up in sending the current through a large external resistance. In this case the difference between the sparking potential and the potential required to maintain the current was 253 volts.

\section{Properties of pointed and cylindrical electrodes.}

When electrodes of different shapes are used, as when a discharge takes place from a point to a plane, the sparking potential depends on the direction of the force.

For pressures above the critical pressure the smaller potential is obtained when the point is negatively charged, and for pressures below a certain value the smaller potential corresponds to the opposite direction of the electric force.

Thus the variation of potential with the pressure may be represented by a curve similar to $P Q R$ when the point is positive, and when the force is reversed the corresponding curve has a relative position similar to $\mathrm{P}^{\prime} Q \mathrm{R}^{\prime}$. The two curves intersect near the point corresponding to the minimum sparking potential, which varies according to the shape of the electrodes.

' Philosophical Maqazine, December, 1904, 
Exactly similar phenomena are observed when the discharge takes place through a gas in the space between two coaxial cylindrical electrodes: for pressures above the critical pressure it is easier to produce a spark when the inner cylinder is negative, and for lower pressures the smaller sparking potential is obtained when the inner cylinder is positively charged.

23. Condition for sparking in a uniform electric field.

The sparking potential between parallel plate electrodes can be deduced immediately from the formula

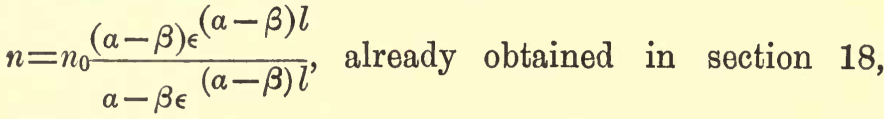
which represents the number of ions $n$ reaching the positive electrode when $n_{0}$ negative ions are started by ultra-violet light from the negative electrode. The quantity $n$ increases as the distance $l$ between the plates increases, the force $\mathrm{X}$ being maintained at a constant value, and when $\beta \epsilon^{(\alpha-\beta) l}$ becomes equal to $a$ the denominator of the above fraction vanishes, and $n$ becomes infinite. A current will therefore continue to flow for an indefinite time after the light ceases to act when the distance between the plates attains the value $\mathrm{S}$ given by the equation

$$
a-\beta \epsilon^{(a-\beta) \mathrm{S}}=0 .
$$

Thus the sparking potential $\mathrm{S} X$ is known for the particular distance $\mathrm{S}$ and pressure $p$ of the gas.

In order to test the theory experimentally, the sparking distances $\mathrm{S}$ corresponding to given values of $\mathrm{X}$ and $p$ were found by means of the formula $S=\frac{1}{a-\beta} \log \left(\frac{a}{\beta}\right)$ 
$a$ and $\beta$ having been found in terms of $\mathrm{X}$ and $p$ by the methods that have been described.

The plates were then set at the exact distances apart $S$, and the potential $\mathrm{V}$ required to produce a spark was determined. The results of the experiments ${ }^{1}$ made with air, hydrogen, nitrogen, carbon dioxide, argon, and helium are given in Tables XVI., XVII., XVIII., XIX., XX., and $\mathrm{XXI}$, and they show the close agreement that exists between the theoretical sparking potential SX and the sparking potential $\mathrm{V}$ determined experimentally. The product of the pressure $p$ and distance $\mathrm{S}$ is given in the last column of each table to illustrate Paschen's law.

\section{TABLE XVI.}

AIR.-Sparking Potentials.

\begin{tabular}{|c|c|c|c|c|c|}
\hline $\mathrm{X}$ & $p$ & $\mathrm{~S}$ & $\mathrm{SX}$ & $\mathrm{V}$ & $p \mathrm{~S}$ \\
\hline & & & & & \\
\hline 1050 & 8 & $\cdot 765$ & 803 & 803 & $6 \cdot 12$ \\
1400 & 8 & $\cdot 431$ & 601 & 603 & $3 \cdot 45$ \\
1050 & 6 & $\cdot 572$ & 601 & 604 & $3 \cdot 43$ \\
700 & 4 & $\cdot 871$ & 610 & 615 & $3 \cdot 48$ \\
1050 & 4 & $\cdot 454$ & 477 & 480 & $1 \cdot 82$ \\
525 & 2 & $\cdot 91$ & 481 & 488 & $1 \cdot 82$ \\
700 & 2 & $\cdot 575$ & 403 & 407 & $1 \cdot 15$ \\
350 & 1 & $1 \cdot 13$ & 395 & 398 & $1 \cdot 13$ \\
437 & 1 & $\cdot 832$ & 364 & 365 & $\cdot 83$ \\
350 & $\cdot 66$ & $\cdot 965$ & 338 & 340 & $\cdot 64$ \\
437 & $\cdot 66$ & $\cdot 766$ & 335 & 336 & $\cdot 505$ \\
& & & & & \\
\hline
\end{tabular}

1 The sparking potentials for air and hydrogen are taken from the papers by the author, Philosophical Magazine, November, 1903, and by the author and Mr. Hurst, Philosophical Magazine, December, 1904 ; those for carbon dioxide and nitrogen are taken from the paper by Mr. Hurst, Philosophical Magazine, April, 1906; and those for argon and helium from the paper by Messrs. E. W. B. Gill and F. B. Pidduck, Philosophical Magazine, August, 1908. 
Sparking Potential in a Uniform Electric Field 57

\section{TABLE XVII.}

Hydrogen.--Sparking Potentials.

\begin{tabular}{|c|c|c|c|c|c|}
\hline $\mathbf{X}$ & $p$ & $\mathbf{S}$ & $\mathbf{S X}$ & $\mathrm{V}$ & $p \mathrm{~S}$ \\
\hline & & & & & \\
\hline & & & & & \\
\hline 1050 & 20 & $\cdot 643$ & 675 & 675 & $12 \cdot 9$ \\
875 & 16 & $\cdot 710$ & 621 & 619 & $11 \cdot 4$ \\
1050 & 16 & $\cdot 463$ & 485 & 490 & $7 \cdot 4$ \\
700 & 12 & $\cdot 791$ & 555 & 561 & $9 \cdot 5$ \\
1050 & 12 & $\cdot 353$ & 370 & 389 & $4 \cdot 23$ \\
25 & 8 & $\cdot 927$ & 486 & 487 & $7 \cdot 42$ \\
700 & 8 & $\cdot 57$ & 399 & 395 & $4 \cdot 56$ \\
050 & 8 & $\cdot 306$ & 322 & 322 & $2 \cdot 45$ \\
350 & 4 & $1 \cdot 14$ & 399 & 395 & $4 \cdot 56$ \\
525 & 4 & $\cdot 613$ & 322 & 323 & $2 \cdot 45$ \\
700 & 4 & $\cdot 405$ & 283 & 282 & $1 \cdot 62$ \\
350 & 2 & $\cdot 810$ & 283 & 287 & $1 \cdot 62$ \\
525 & 2 & $\cdot 501$ & 269 & 273 & $1 \cdot 00$ \\
350 & 1 & $\cdot 806$ & 282 & 289 & $\cdot 81$ \\
& & & & & \\
\hline
\end{tabular}

\section{TABLE XVIII.}

Nitrogen.--Sparking Potentials.

\begin{tabular}{|c|c|c|c|c|c|}
\hline $\mathrm{X}$ & $p$ & $\mathrm{~S}$ & SX & $\mathrm{V}$ & $p \mathbf{S}$ \\
\hline \multirow[t]{3}{*}{700} & 4 & $\cdot 72$ & \multirow[t]{3}{*}{502} & 507 & $2 \cdot 88$ \\
\hline & 4 & $\cdot 6$ & & 460 & $2 \cdot 4$ \\
\hline & 2 & $\cdot 85$ & & 382 & $1 \cdot 70$ \\
\hline \multirow{6}{*}{525} & 2 & $\cdot 665$ & \multirow[t]{6}{*}{349} & 344 & $1 \cdot 33$ \\
\hline & 2 & $\cdot 65$ & & 339 & $1 \cdot 30$ \\
\hline & 2 & $\cdot 58$ & & 332 & $1 \cdot 16$ \\
\hline & $\overrightarrow{1}$ & 1.0 & & 310 & 1.00 \\
\hline & 1 & $\cdot 868$ & & 300 & $\cdot 868$ \\
\hline & 1 & $\cdot 601$ & & 298 & $\cdot 601$ \\
\hline \multirow{3}{*}{525} & 1 & $\cdot 58$ & \multirow[t]{3}{*}{304} & 300 & $\cdot 58$ \\
\hline & 1 & $\cdot 5$ & & 310 & $\cdot 5$ \\
\hline & 1 & $\cdot 4$ & & 330 & $\cdot 4$ \\
\hline
\end{tabular}


Table XIX.

Carbon Dioxide.-Sparking Potentials.

\begin{tabular}{|c|c|c|c|c|c|}
\hline $\mathrm{X}$ & $p$ & $\mathrm{~S}$ & $\mathrm{SX}$ & $\mathrm{V}$ & $p \mathrm{~S}$ \\
\cline { 1 - 2 } 1400 & 2 & $\cdot 369$ & 516 & 517 & $\cdot 738$ \\
700 & 1 & $\cdot 736$ & 516 & 509 & $\cdot 736$ \\
875 & 1 & $\cdot 571$ & 500 & 495 & $\cdot 571$ \\
1050 & 1 & $\cdot 465$ & 488 & 491 & $\cdot 465$ \\
525 & $\cdot 5$ & $\cdot 929$ & 488 & 485 & $\cdot 464$ \\
700 & $\cdot 5$ & $\cdot 706$ & 494 & 497 & $\cdot 353$ \\
875 & $\cdot 5$ & $\cdot 613$ & 537 & 530 & $\cdot 306$ \\
525 & $\cdot 25$ & $1 \cdot 06$ & 558 & 564 & $\cdot 265$ \\
\hline
\end{tabular}

TABLE XX.

Argon.-Sparking Potentials.

\begin{tabular}{|c|c|c|c|c|c|}
\hline $\mathrm{X}$ & \multirow{2}{*}{$p$} & $\mathrm{~S}$ & $\mathrm{SX}$ & $\mathrm{V}$ & $p \mathrm{~S}$ \\
\cline { 1 - 1 } 505 & 10 & $1 \cdot 087$ & 549 & 549 & $10 \cdot 87$ \\
610 & 4 & $\cdot 471$ & 287 & 276 & $1 \cdot 88$ \\
410 & 4 & $\cdot 947$ & 388 & 380 & $3 \cdot 79$ \\
618 & 2 & $\cdot 378$ & 234 & 233 & $\cdot 76$ \\
401 & 2 & $\cdot 618$ & 248 & 245 & $1 \cdot 24$ \\
615 & 1 & $\cdot 399$ & 245 & 248 & $\cdot 399$ \\
517 & 1 & $\cdot 482$ & 249 & 244 & $\cdot 482$ \\
401 & 1 & $\cdot 580$ & 233 & 235 & $\cdot 580$ \\
309 & 1 & $\cdot 770$ & 248 & 237 & $\cdot 770$ \\
402 & $\cdot 66$ & $\cdot 599$ & 241 & 248 & $\cdot 395$ \\
301 & $\cdot 66$ & $\cdot 802$ & 242 & 238 & $\cdot 528$ \\
\cline { 2 - 4 } & & & & & \\
\hline
\end{tabular}


Sparking Potential in a Uniform Electric Field 59

TABLE XXI.

HELIUM (before purification with liquid air). ${ }^{1}$ —Sparking Potentials

\begin{tabular}{|c|r|c|c|c|c|}
\hline $\mathrm{X}$ & $p$ & $\cdot \mathrm{S}$ & $\mathrm{SX}$ & $\mathrm{V}$ & $p \mathrm{~S}$ \\
\hline & & & & & \\
\cline { 2 - 5 } 404 & 13 & $\cdot 674$ & 272 & - & $8 \cdot 76$ \\
305 & 8 & $\cdot 845$ & 258 & 262 & $6 \cdot 76$ \\
385 & 10 & $\cdot 650$ & 250 & 252 & $6 \cdot 50$ \\
305 & 6 & $\cdot 781$ & 238 & 236 & $4 \cdot 69$ \\
304 & 4 & $\cdot 760$ & 231 & 234 & $3 \cdot 04$ \\
506 & 6 & $\cdot 462$ & 234 & 232 & $2 \cdot 77$ \\
772 & 6 & $\cdot 326$ & 254 & 246 & $1 \cdot 96$ \\
304 & 2 & $\cdot 863$ & 262 & 262 & $1 \cdot 73$ \\
402 & 2 & $\cdot 694$ & 279 & 274 & $1 \cdot 39$ \\
\hline
\end{tabular}

The sparking potentials in volts are also given in the following curves, figure 11, the abscissæ being the product of the pressure and the distance between the plates.

The accuracy of the theory is thus established over a range of pressures extending from the critical pressure to a pressure twelve times as great for any distance between the plates. The theory has not been tested for pressures below the critical pressure, as the values of $a$ and $\beta$ were not determined for the lower pressures for the reasons already explained.

It may readily be seen from the theory that the potential SX is a function of $p \mathrm{~S}$ only, and consequently that Paschen's law is satisfied. For if $v$ denotes the theoretical sparking potential SX and $m$ the product $p \mathrm{~S}$ which is proportional to the quantity of gas between the electrodes, then $a=p f\left(\frac{\mathrm{X}}{p}\right)=p f\left(\frac{v}{m}\right)$,

1 The sparking potentials for pure helium are much lower; the minimum does not exceed 200 volts. 
which sparking took place when ultra-violet light of small intensity was acting on the negative electrodes. The potentials thus obtained were very definite, and showed no irregularities, being independent of the length of time during which the gas is subjected to the electric force. They were in most cases about two volts below the potential required to spark when no light is acting on the negative electrode.

The lowering of the sparking potential due to the action of ultra-violet light, which was discovered by Hertz, ${ }^{1}$ is more noticeable when the light is strong and the pressure high. According to this theory, it is due to

the large current proportional to $n=n_{0} \frac{(a-\beta) \epsilon^{(a-\beta) l}}{a-\beta \epsilon}{ }^{(a-\beta) l}$ which ensues when $\mathrm{X}$ and $n_{0}$ are large, before the denominator of the fraction vanishes. The polarization due to the separation of the positive and negative ions disturbs the uniformity of the field between the plates in such a way as to facilitate the discharge, as will be explained in section 27. The charge in the gas which causes the polarization is proportional to the intensity of the light, and inversely proportional to the velocity of the ions. This latter quantity diminishes as the pressure of the gas between the plates increases, since $\mathrm{X} / p$ for sparking diminishes as the quantity $p \mathrm{~S}$ rises. It is therefore necessary to use ultra-violet light of very small intensity in order to determine with accuracy the sparking potential which corresponds to the theoretical value derived from the equation $a-\beta \epsilon^{(a-\beta) \mathrm{S}}=0$.

When no light is falling on the electrodes the sparking

1 Hertz, Wied Ann., xxxi., 1887. 
is due to the multiplication of the few ions which under all conditions are present in the gas. Theoretically any small number of ions would be sufficient to initiate a discharge when the plates are at the sparking distance, $\mathrm{S}$ corresponding to the electric force, and that a few ions are being continually generated in a gas even when it is contained in a closed vessel has been shown by Geitel $^{1}$ and C. T. R. Wilson. ${ }^{2}$ But the number of such ions is very small, and being generated throughout the volume of the gas, they are not on the average as efficient for starting a spark as ions coming from the negative electrode, each of which traverses the whole distance between the electrodes. This accounts for the small difference of two or three volts between the ordinary sparking potential and that obtained when ultra-violet light of small intensity falls on the negative electrode.

1 Geitel, Physikalische Zeitschr., ii., 1900.

2 C. T. R. Wilson, Cam. Phil. Soc., xi., 1900. 


\section{CHAPTER IV}

THEORY OF ELECTRIC DISCHARGES IN FIELDS OF FORCE WHICH ARE NOT UNIFORM

25. Description of phenomena to be investigated.

The theory may also be applied to discharges between electrodes in gases in which the electric force varies from point to point along the path of the current; but in these cases the investigations cannot be carried out so completely as those which deal with the sparking potential in a uniform field between two parallel plates. Thus the potential required to produce a point discharge cannot be expressed in a form that admits of being compared with experimental results; but it can be shown from the theory that the potential is smaller when the point is negative than when the point is positive throughout the range of forces and pressures for which $a$ and $\beta$ have been determined. Another case which is of interest is the potential required to maintain a current between two parallel plates. When the current is small the uniformity of the field is not appreciably disturbed by the charge in the gas, so that the potential required to maintain the current is the same as the sparking potential. But when the current increases the charge in the gas increases the force near the negative electrode, and under these circumstances it can be shown that the potential between the electrodes which would maintain the current is less than the 
sparking potential. The difference between these two potentials arises from the same effects as produce the difference between the sparking potentials for negative and positive points.

It is necessary to point out that there are limits to the ranges of values of the current, electric force, and pressure for which the collision theory, as it is here discussed in a simple form, can be expected to give a complete explanation of the experimental results. When the current is large the gas becomes heated, and the number of molecules in the path of the discharge is diminished; also a certain proportion of the positive and negative ions recombine, and this introduces a further complication. When the pressures are very low, and large forces are required to produce a discharge, radiations, such as cathode rays and Röntgen rays, are emitted, which contribute to the formation of ions and thus facilitate the discharge. A comparison of the sparking potentials for cylinders and parallel plates shows that radiation effects are probably of importance when the pressure is somewhat below the critical pressure. The investigations will therefore be confined at first to pressures above the critical pressure and to the smaller currents, for which it is highly improbable that there are any other processes of ionization taking place that add appreciably to the number of ions generated by collisions.

In order to examine the experimental results, and to see how far they may be explained by the theory, it is necessary to find the condition that a given field of force should suffice to maintain a current when the supply of ions is kept up by the effects of collisions. An expression may be found for this condition in the general case by 
a method similar to that which was used to determine the condition for sparking in a uniform field, but it is interesting to investigate the problem on somewhat different principles. ${ }^{1}$

26. Condition for the maintenance of a current by effects of collisions in any field of force.

Let the current pass between two parallel plate electrodes at a distance $l$ apart, and let $x$ be the distance of any point in the gas from the negative electrode. Let $u$ be the velocity of the negative ions, $a$ the number of molecules ionized by a negative ion in travelling through a centimetre of the gas, $n_{1}$ the number of negative ions per cubic centimetre, $v, \beta$, and $n_{2}$ similar quantities for positive ions. These quantities depend on the force $\mathrm{X}$, which is supposed to vary from point to point. If $e$ is the charge on an ion, then $e\left(n_{1} u+n_{2} v\right)$ represents the current in the gas, so that $n_{1} u+n_{2} v=c$ is constant along the path of the discharge.

When the current is steady there are no variations with respect to the time at any point in the gas, so that

$$
\frac{d n_{1}}{d t}=-\frac{d}{d x}\left(n_{1} u\right)+a n_{1} u+\beta n_{2} v=0,
$$

and

$$
\frac{d n_{2}}{d t}=-\frac{d}{d x}\left(n_{2} v\right)+a n_{1} u+\beta n_{2} v=0 .
$$

Hence

$$
\frac{d}{d x}\left(n_{1} u\right)=a n_{1} u+\beta n_{2} v=(\alpha-\beta) n_{1} u+\beta c,
$$

so that

$$
n_{1} u=\operatorname{R\epsilon }^{\int(\alpha-\beta) d x}{ }_{+\epsilon}^{\int(\alpha-\beta) d x} \int_{\beta c \epsilon}-\int(\alpha-\beta) d x d x
$$

${ }^{1}$ See papers by the author, Philosophical Magazine, March, 1905, and June, 1906. 
Let $\mathrm{Z}(x)$ denote the quantity $\int_{0}^{x}(a-\beta) d x$; thes may be expressed in the form

and similarly

$$
n_{1} u=\mathrm{AZ}(x)+c \mathrm{Z}(x) \int_{0}^{x}[\mathrm{Z}(x)]^{-1} \beta d x,
$$

$$
n_{2} v=\mathrm{BZ}(x)-c \mathrm{Z}(x) \int_{0}^{x}[\mathrm{Z} x]^{-1} a d x .
$$

Since all the ions are produced in the gas, the follo conditions hold at the electrodes :-

At the negative electrode $x=0, n_{1}=0$, and $n_{2} v=c$.

At the positive electrode $x=l, n_{2}=0$, and $n_{1} u=c$.

The first two conditions give $\mathrm{A}=0$ and $\mathrm{B}=c$, anc second two give

$$
\begin{gathered}
1=\mathrm{Z}(l) \int_{0}^{l}[\mathrm{Z}(x)]^{-1} \times \beta d x, \text { and } \\
1=\int_{0}^{l}[\mathrm{Z}(x)]^{-1} \times a d x,
\end{gathered}
$$

which are not independent, since

$$
\int_{0}^{x}(\beta-a)[\mathrm{Z}(x)]^{-1} d x=[\mathrm{Z}(x)]^{-1}-1,
$$

so that the equation

$$
\mathbf{1}=\int_{0}^{l} a \epsilon \int_{0}^{x}(\beta-a) d x d x
$$

represents the condition which must be satisfied by values of $a$ and $\beta$ along the path from the neg: electrode $x=0$ to the positive electrode $x=l$ in o that a continuous current should be maintained.

When the current is very small, so that the fiel force between the plates is uniform, $a$ and $\beta$ are cons and the above condition reduces to

$$
1=\frac{a}{\beta-a}\left(\epsilon^{(\beta-a) l}-1\right) \text {, or } a=\beta \epsilon^{(a-\beta) l,}
$$


which is the equation that determines the sparking potential. Hence the sparking potential may be defined as the potential which is required to maintain a very small current in the gas.

27. Currents accompanied by a positive charge in the gas.

In order to investigate the effect of the increase of current on the potential, it is necessary to consider how the force between the plates is affected by the electricity in the gas. The positive ions, being of larger mass, travel more slowly than the negative ions, so that there is a greater number of positive than of negative ions in the gas at any time. This excess of positive electricity causes an appreciable disturbance in the uniformity of the field as the current rises, and since all the positive ions must pass through the gas at the negative electrode, the positive charge is greatest in the neighbourhood of that electrode. The effect of this charge is to increase the force near the negative electrode and to diminish it in other parts of the field. The ionizing power of the positive ions is much increased in the field of strong electric force, and when that field is near the negative electrode the supply of ions is kept up when the potential difference between the electrodes is less than the sparking potential.

Experiments on currents through rarefied gases in discharge tubes show that the force is greater near the cathode than at other points of the discharge. The phenomena which attend these discharges are complicated, and have given rise to numerous speculations, but the principal variation in the electric force was first explained by Professor Schuster ${ }^{1}$ in the manner given

1 Bakerian Lecture, Proc. Roy. Soc., xlvii., 1890, p. 541. 
above. He assumed that the positive and negative ions in the gas have equal charges, the same as the atomic charges on ions in liquid electrolytes, a hypothesis which subsequent experiments have confirmed, and attributed the positive charge in the gas to the difference in mass between the positive and negative ions.

28. Increase of force at cathode accompanied by decrease of potential required to maintain a current between the electrodes for pressures above the critical pressure.

The decrease in the potential difference between the electrodes that accompanies the increase of the electric force at the cathode may also be determined theoretically by finding the potential required to maintain a current in a field made up of two parts in each of which the force is constant, the value of the constant being greater in the part near the negative electrode.

Let $a$ and $\beta$ have the values $a_{1}$ and $\beta_{1}$, corresponding to a force $\mathrm{X}_{1}$, through a space of thickness $a$ on the side of the cathode, and the values $a_{2}$ and $\beta_{2}$, corresponding to a force $\mathrm{X}_{2}$, in the rest of the field of thickness $b$.

$$
\begin{array}{c|c:c|c}
\begin{array}{c}
\text { Negative } \\
\text { electrode } \\
x=0
\end{array} & a_{1} \beta_{1} & a_{2} \beta_{2} & \begin{array}{c}
\text { Positive } \\
\text { electrode } \\
x=l .
\end{array} \\
\mathrm{X}_{1} & \mathrm{X}_{2} &
\end{array}
$$

In this case the condition for the maintenance of the current between the plates becomes

$$
{\frac{a_{1}-\beta_{1} \epsilon}{a_{1}-\beta_{1}}}^{\left(a_{1}-\beta_{1}\right) a}+\frac{\beta_{2}-a_{2} \epsilon}{\beta_{2}-a_{2}}{ }^{\left(\beta_{2}-a_{2}\right) b}=1 .
$$

In order to apply this formula to a definite case, let the gas between the electrodes be hydrogen at 10 millimetres 
pressure, and let a force of 80 volts per millimetre act in a layer extending 2 millimetres from the cathode, and a force of 50 volts per millimetre in the rest of the field. The values of $a_{1}, \beta_{1}, a_{2}, \beta_{2}$, as obtained from the curves giving $\alpha / p$ and $\beta / p$ in terms of $\mathrm{X} / p$, are as follows :

$$
a_{1}=10 \cdot 0, \beta_{1}=\cdot 081, \alpha_{2}=3 \cdot 6, \beta_{2}=\cdot 021 \text {, }
$$

so that the distance $b$ may be found from the above equation, since $a$ is given equal to $\cdot 2$. The value of $b$ thus determined is 8 millimetres, and hence the total fall of potential between the electrodes is $80 \times 2+50 \times 8$ $=560$ volts, which is about 20 volts less than the sparking potential in hydrogen at 10 millimetres pressure between plates a centimetre apart.

Larger differences between the potentials are obtained when further increases are made in the force near the cathode. Thus, if $X_{1}=200$ volts per millimetre in the first millimetre of gas near the cathode and 40 volts per millimetre in the rest of the field, a similar calculation gives $b=3 \cdot 25$, so that the fall of potential between the electrodes is 330 volts, the corresponding sparking potential between the electrodes, 4.25 millimetres apart, being 385 volts. It is thus obvious, from the theoretical results that have been obtained, that the potential required to maintain the current in the gas diminishes as the current increases, owing to the increase of the force near the negative electrode.

\section{Simple experiments to illustrate the effect of concentrating the force near the cathode.}

These results are in accordance with the experimental investigations ${ }^{1}$ for pressures above the critical

${ }_{1}$ Philosophical Magazine, June, 1906. 
pressure, as may be seen from the following experiments with air. Instead of depending on the charge on the positive ions to intensify the field near the negative electrode, a gauze may be used near the negative electrode and maintained at any required potential.

Two electrodes, A and B, were set up, as shown in the accompanying diagram, figure 12 , in an air-tight glass tube, and a grating of fine wire $G$ was placed near the negative electrode, and could be charged to any potential

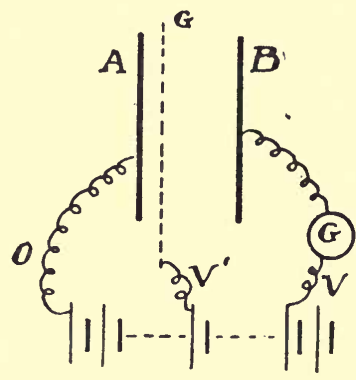

Figure 12.

$\mathrm{V}^{\prime}$ intermediate between those of $\mathrm{A}$ and $\mathrm{B}$ by means of a separate connection. The sparking potential between $\mathrm{A}$ and $B$ was then found to depend on the potential of the grating. The following results ${ }^{1}$ were obtained, in which $V^{\prime}$ represents the potential difference between the grating and the negative electrode and $\mathrm{V}$ the sparking potential between $\mathrm{A}$ and $\mathrm{B}$ :-

\begin{tabular}{|c|c|c|c|c|c|}
\hline $\mathrm{V}^{\prime}$ & 100 & 150 & 170 & 210 & 240 \\
\hline $\mathrm{V}$ & 700 & 680 & 640 & 600 & $\tilde{5} 50$ \\
\hline
\end{tabular}

1 Philosophical Magazine, June, 1906. 
Thus the sparking potential between A and B was lowered by 150 volts by concentrating the force near the cathode.

The essential points on which this theory of the diminution of the potential required to maintain a current below the sparking potential is based may be stated briefly as follows:-

The supply of ions by which the current is maintained depends chiefly on the negative ions, and of these the most efficient are those generated near the negative electrode, since they travel nearly the whole length of the discharge. When the force is increased near that electrode and the total fall of potential between the electrodes is diminished, two effects are produced: the number of ions generated by one of the negative ions in travelling between the electrodes is diminished, and the number of ions generated by a positive ion near the negative electrode is increased, thus increasing the number of the most active negative ions. Now the rate of increase of $\beta$ with the force $X$ bears to $\beta$ a much greater ratio than the corresponding rate of increase of $a$ bears

to $a\left[\operatorname{or} \frac{1}{\beta} \frac{d \beta}{d \mathrm{X}}>\frac{1}{a} \frac{d a}{d \mathrm{X}}\right]$ and on this depends the maintenance of the current in spite of the large diminution which takes place in the potential difference between the electrodes. When this potential is reduced the average value of $a$ along the path of the discharge is diminished, and the effect of the negative ions, which depends on an

exponential term (such as $\mathrm{N}_{0} \epsilon^{a \mathrm{~S}}$ ), is greatly reduced; but, owing to the large increase in the value of $\beta$, a compensating effect of the same order is introduced (in 
the quantity $\mathrm{N}_{0}$ ) by the increased activity of the positive ions at the negative electrode.

30. Comparison of the effects of concentrating the force near the cathode and near the anode; explanation of sparking potentials for positive and negative points.

It is interesting to examine the equation

$$
{\frac{a_{1}-\beta_{1} \epsilon}{a_{1}-\beta_{1}}}^{\left(a_{1}-\beta_{1}\right) a}+{\frac{\beta_{2}-a_{2} \epsilon}{\beta_{2}-a_{2}}}^{\left(\beta_{2}-a_{2}\right) b}=1
$$

more generally and to contrast the effects of increasing the force in the parts of the field near the cathode and near the anode.

For a field of force chosen arbitrarily the sum of the two fractions in the above equation has a value $\mathrm{Y}$ which is different from unity. When the values of $a$ and $\beta$ are small, the forces do not suffice to maintain a current, and each of the fractions becomes equal to unity, so that $\mathrm{Y}=2$. As the values of $a$ and $\beta$ increase the fractions diminish, and their sum becomes equal to unity when the forces are sufficient to maintain a discharge. Hence, when the value of $\mathrm{Y}$ lies between 1 and 2 for a system of values of $a$ and $\beta$, the forces between the electrodes will not suffice to maintain a current.

The effect of reversing a field may be found by interchanging $a_{1}$ and $\beta_{1}$, and $a_{2}$ and $\beta_{2}$, and if this be done in the two numerical examples given above, it will be found that $\mathrm{Y}$ is greater than unity in each case, so that when the forces are reversed they are not sufficient to maintain a current.

A very simple case arises when one of the forces $\mathrm{X}_{2}$, acting through the distance $b$, is so small that the 
corresponding value of $\beta_{2}$ may be neglected. If $\mathrm{X}_{2}$ be on the side of the negative electrode, the condition for the maintenance of a current is

$$
\frac{\beta_{1}-a_{1} \epsilon^{\left(\beta_{1}-a_{1}\right) a}}{\beta_{1}-\alpha_{1}}+\frac{a_{2}}{a_{2}}=1,
$$

which shows that $a_{1}-\beta_{1} \epsilon^{\left(a_{1}-\beta_{1}\right) a}=0$, or that the potential fall in the distance $a$ on the positive side must be the sparking potential $\mathrm{V}_{a}$ for parallel plates at the distance $a$ apart. Hence the potential difference between the electrodes is in this case

$$
\mathrm{V}_{a}+\mathrm{X}_{2} b \text {. }
$$

If the forces are reversed so that $\mathrm{X}_{2}$ is on the side of the positive electrode, the condition for the maintenance of a current reduces to

$$
\frac{a_{1}-\beta_{1} \epsilon^{\left(a_{1}-\beta_{1}\right) a}}{a_{1}-\beta_{1}}+\epsilon^{-a_{2} b}=1,
$$

which shows that $a_{1}$ must exceed $\beta_{1} \epsilon^{\left(a_{1}-\beta_{1}\right) a}$, and consequently the potential fall along the distance $a$ is less than the sparking potential $\mathrm{V}_{\alpha}$, so that the potential difference between the electrodes must be less than $\mathrm{X}_{2} b+\mathrm{V} a$.

The most familiar examples of phenomena which may be explained on this principle are point discharges. When the point is negative, the strong field is near the negative electrode, so that the potential required to produce a discharge is less than when the point is positive. The same result is obtained when a gas is contained between two concentric cylinders: the lower 
sparking potential corresponds to the case in which the inner cylinder is negative.

The theory thus gives a satisfactory explanation of the variation of the potential with the current for parallel plate electrodes and also of the difference between the sparking potentials for point discharges when the direction of the force is reversed.

\section{Phenomena at pressures below the critical pressure.}

The preceding results can only be considered to hold for pressures above the critical pressure, as the experiments by which the values of $a$ and $\beta$ were determined show that for these pressures the ionization of the gas is completely accounted for by the action of the positive and negative ions in generating others by collisions with molecules. The curves which have been obtained for the values of $a$ and $\beta$ show that $a / p$ does not vary much with the force for large values of $\mathrm{X} / p$, so that $a$ diminishes nearly in direct proportion to the pressure when the force is large and the pressure is below the critical pressure. The question then arises whether the quantity $\beta$ increases sufficiently rapidly to account for the sparking potentials obtained experimentally for the low pressures. It is easy to calculate roughly the values of $\beta$ which are necessary, and they do not appear to be greater than the numbers that might be expected to correspond to points on continuations of the curves for $\beta / p$, but the question cannot be decided in this way, as it is difficult to apply the theory of collisions accurately when the free paths of the ions become considerable fractions of the distance between the plates. Some evidence of the processes which influence the sparking at the low pressures may, however, be obtained 
by examining either the variations of the potential difference between the plates for different currents or the sparking potentials between conductors arranged so as to produce a variation of the force along the path of the discharge.

If the supply of ions were maintained by the effects of collisions at the lower pressures, the sparking potential for a gas between parallel plates should continue to be greater than the potential difference which maintains a current, but experiments show that at the lower pressures the potential increases with the current. ${ }^{1}$ This might be due to a rise of temperature of the gas and a corresponding reduction in the number of molecules between the plates, in which case the potential maintaining the current would tend to increase in the same way as the sparking potential increases when the pressure diminishes. This suggestion has not yet been tested experimentally, so that it is uncertain whether it affords a complete explanation of the effects obtained.

These phenomena are most probably related to the point discharges for the lower pressures, and a theory which would explain why sparking is produced more easily from a positively charged point in a gas at a low pressure than from the same point negatively charged would apply also in some degree to the potential required to maintain a current.

32. Processes of ionization that may account for effects obtained at low pressures.

In these cases, as well as in the discharge between cylinders, the lower sparking potential is obtained when the greater force in the gas is in the neighbourhood of

I J. A. Brown, Philosophical Magazine, September, 1906. 
the positive electrode. It would appear from this that some new process of ionization is called into play which acts in addition to the effects of collisions. The force in the neighbourhood of the positive electrode affects principally the negative ions, all of which pass through that region. The potentials which are obtained experimentally can therefore be explained on the hypothesis that some form of radiation is emitted from the anode when the negative ions impinge on it. Some such effect might be expected, as it is known that Röntgen rays are produced by the same cause when the pressure is very low. The pressures under consideration are, however, large compared with any pressure in a vacuum tube at which Röntgen rays can be detected outside the tube by ordinary methods, but it is possible that non-penetrating rays of a similar kind may be produced, capable of ionizing the gas inside the tube, at these comparatively high pressures. The experimental results may thus be explained in a general way, but it is impossible at this stage to form a complete theory of the sparking potentials at pressures below the critical pressure, as they have not yet been examined very extensively with a view to obtaining evidence as to the relative importance of the processes of ionization that take place.

It may be pointed out, in connection with the possible existence of radiations of a non-penetrating character inside a discharge tube, that $\mathrm{E}$. Wiedemann ${ }^{1}$ has found that, when a current is established in a gas, easily absorbed rays (termed Entladungstrahlen) are emitted by the luminous portions of the discharge, and Professor Sir J. J. Thomson has shown that these rays have the

1 Wied. Ann., lx., p. 269. 
property of ionizing the gas. ${ }^{1}$ A radiation of this kind would obviously affect the potential required to maintain a current, but if the intensity of the radiation is proportional to the square of the number of ions per cubic centimetre of the discharge, as Professor H. A. Wilson's ${ }^{2}$ investigations on the ionization in the positive column seem to indicate, the rays would be inappreciable with small currents, and would not affect sparking potentials.

33. Cathode fall of potential; ionization in the space near the cathode when the cathode fall of potential is established.

In gases above the critical pressure the currents when large are accompanied by the phenomena known as the cathode fall of potential. The polarization in the gas as measured by this fall does not vary with the current, and the potential difference between the electrodes also remains approximately constant. At this stage the effects which take place in the gas are so complicated, involving recombination and various changes in the velocities of the ions, that no theory has been proposed which explains the distribution of force in the path of the discharge and accounts for the remarkable fact that certain potentials are almost independent of the current. It is, however, easy to show in a general way that the ionization arising from collisions in these cases may be sufficient to maintain the current.

The condition which must be satisfied by the values of $a$ and $\beta$ along the path of the discharge between electrodes at a distance $l$ apart may be written in the form

1 J. J. Thomson, Proceedings Cambridge Philosophical Society, Vol. x., Pt. ii., p. 74.

2 Philosophical Magazine, July, 1903. 


$$
1-\int_{0}^{b} a \epsilon \int_{0}^{x}(\beta-a) d x d x=\int_{b}^{l} a \epsilon \int_{0}^{x}(\beta-a) d x d x,
$$

and if the values of $a$ and $\beta$ are very small in the column of gas of length $l-b$ in contact with the positive electrode, the quantity on the right of the above equation becomes very small, and the integral

$$
\int_{0}^{b} a \epsilon \int_{0}^{x}(\beta-a) d x d x
$$

becomes nearly equal to unity, so that the potential fall along the distance $b$ (from $x=0$ to $x=b$ ) at the negative electrode is nearly the same as the potential that would maintain the same current between electrodes at a distance $b$ apart, the pressure of the gas being unchanged.

It is possible to show the connection between the cathode fall of potential and the minimum sparking potential by using the above investigation in connection with the following experimental results.

The sparking potential has a minimum value corresponding to a certain amount of gas between the plates, which may be measured by the product $p b$, where $p$. is the pressure and $b$ the critical distance between the plates for the pressure $p$. In this case the sparking potential does not differ much from the potential required to maintain a discharge in the gas. Thus in a set of experiments with hydrogen at a pressure of 2.55 millimetres, the following potentials were obtained between electrodes 44 centimetres apart: 273 volts for a very small current and 272,255 , and 250 volts for currents of $2 \times 10^{-5} 2 \times 10^{-4}$ and $4.6 \times 10^{-3}$ ampères respectively. Within an error of about 4 per cent. the 
value of the potential may be taken as 260 volts for any of the currents.

If, therefore, the plates are at a large distance apart and the fall of potential along a layer of thickness $b$ near the negative electrode has attained the value of 260 volts, $b$ being the distance between electrodes for which the pressure in the gas is the critical pressure, the current will be maintained by the ions generated in that layer, even when the force in the rest of the discharge is not sufficient to cause ions to be generated by collision. The experiment quoted shows that the potential fall along the distance $b$ need not vary much with the current.

Thus the cathode fall at a given pressure $p$ should be independent of the current and of the distance between the electrodes, should be equal to the minimum sparking potential, and should extend over a distance $b$, where $p \times b$ is a measure of the amount of gas corresponding to the minimum sparking potential, and $b$ should consequently vary inversely as $p$. These conclusions are in general agreement with experiment.

The cathode fall of potential as determined experimentally is independent of the length of the discharge and the intensity of the current, and has been shown by Strutt ${ }^{1}$ to be practically the same as the minimum sparking potential. Experiments also show that the thickness $b_{1}$ of the layer of gas across which the fall of potential takes place increases as the pressure diminishes, though it cannot be found very accurately as the force is very small at a short distance from the cathode. The product $p b_{1}$, as given by most observers, is less than $p b$,

1 Hon. R. J. Strutt, Philosophical Transactions, Vol. cxciii., p. $377,1900$. 
but since the force is very small along the distance $b-b_{1}$, the experiments show that for a layer of thickness $b$ the potential fall is practically the minimum sparking potential, and the product $p b$ may be taken as constant.

\section{Sparking potential at atmospheric pressure for very short distances between the electrodes.}

Some experiments made by Earhart ${ }^{1}$ dealing with very short spark gaps in air at atmospheric pressure seem to be at variance with the accumulated evidence, which shows that there is a minimum spark potential below which it is impossible to obtain a discharge through a gas. This minimum is about 340 volts for air, but in some cases, when the electrodes were very close together, it was found that a current passed between them when the potential difference was as low as 30 volts. It was at first supposed that the current passed through the air between the electrodes, but recent experiments made by $A l m y^{2}$ show that the electrodes are liable to be drawn together and to come into contact, owing to the large electrostatic force between them when they are a short distance apart and a potential difference is established between them. It was found that when the apparatus was constructed so that the electrodes do not become displaced a potential of 330 volts is not sufficient to produce a discharge through air at atmospheric pressure, while 360 volts is sufficient to do so. This result is in accordance with the theory that has been given for the sparking potential, and establishes the interesting fact

1 Earhart, Philosophical Magazine, 1901, p. 147.

2 J. E. Almy, Philosiphical Magazine, September, 1908. 
that the large forces developed at the surfaces of conductors separated by a fraction of the wave length of sodium light, and differing in potential by 300 volts, are not sufficient to make electricity pass from the conductor to the surrounding gas.

35. Remarks on processes of ionization which account for various phenomena.

In the preceding account of the development of currents in gases it has been shown that the conductivities may be explained on the theory that all the ionization is to be attributed to ions produced in the gas by positive and negative ions. Some of the phenomena that have been discussed might also be fairly well accounted for by supposing that the negative ions ionize the molecules of the gas, and that the positive ions have only the power of setting free negative ions when they impinge on the negative electrode. There are good reasons, however, for preferring the former method of explaining the phenomena that occur at pressures above the critical pressure, and of showing the connection between them. In the first place, if the positive ions acted by setting free negative ions from the cathode, their effect would presumably depend on the metal of the electrode, and the sparking potentials would show variations depending on the metal of which the electrode was made. This point has been examined recently by Carr (loc. cit.), and the results of several careful experiments have shown that the sparking potential is independent of the metal. This conclusion is in agreement with the observations of most other physicists, but some have found that with aluminium, and possibly also with magnesium, the sparking potentials are somewhat lower than with other metals. 
Again, if the case of point discharges be considered it will be seen that when the point is positive the supply of negative ions cannot be kept up by the action of positive ions at the negative electrode. For this electrode might be large and so distant from the point, that the force at its surface would be too small to give the positive ions a velocity of impact sufficient for the production of negative ions. In this case it is obvious that the effect of the positive ions must be attributed to their action on the molecules of the gas, and the difference between the sparking potentials for positive and negative points follows immediately from this theory, as has been explained above. Another fact to be noticed is that a positive ion makes only one collision with the electrode, whereas it makes a large number of collisions with molecules of the gas, and there is no reason for supposing that a negative ion is more easily set free from a metal than from the gas molecules. There is thus definite evidence to show that molecules of the gas are ionized by the positive ions, and for pressures above the critical pressure a consistent explanation may be obtained of a large number of phenomena on the theory that this is the predominating effect produced by the positive ions.

On the other hand, at pressures below the critical pressure the negative ions forming the cathode rays make up an integral part of the current, so that for these low pressures the ions coming from the negative electrode should be taken into consideration, and when they introduce appreciable effects the sparking potentials might be expected to depend on the metal of the electrode. 36. Examination of some other theories of the sparking potential.

Several expressions for the sparking potential in terms of the pressure have been given by physicists, 
some of which are empirical and do not aim at giving an explanation of the fundamental principles on which the potentials depend, and others, depending on various assumptions which attribute certain properties to positive and negative ions, appear to explain more or less accurately some of the phenomena which are observed. Professor Sir J. J. Thomson has recently given two theories of the latter kind, and it is interesting to examine how far they furnish results which account for the various potentials and to what extent the assumptions made in the process of the investigations agree with those properties of ions which are found to accord with simpler phenomena.

The first ${ }^{1}$ of these investigations differs essentially from that given in section 20 above, and deals with the problem of finding the potential required to maintain a current flowing between electrodes when the ordinary cathode fall of potential is established. No distinction is drawn between the sparking potential and the potential required to maintain a current. It is obvious that the expression which is thus found cannot represent the sparking potential, and the apparent agreement between the formula and the sparking potentials obtained experimentally results only from attributing convenient values to undetermined constants. Clearly the formula for the potential that is found in this way should represent the potential required to maintain a comparatively large current, if the assumptions on which the theory is based are correct.

The current on this theory is kept up by the ionization of the molecules of the gas by negative ions, and a

'J. J. Thomson, "Conduction of Electricity through Gases," 1903, p. 38. 
supply of the latter is supposed to be generated at the cathode by the impact against it of positive ions. The expression for the sparking potential $\mathrm{V}$ as found on this hypothesis is

$$
\mathrm{V}=\mathrm{V}_{0}+\frac{1}{\beta e} \log \cdot \frac{\left(1+k \mathrm{~V}_{0} e\right)}{k \mathrm{~V}_{0} e}-\frac{c(1+w)}{\lambda} \frac{1}{\beta e}+\frac{(\gamma+w)}{\beta e} \frac{d}{\lambda},
$$

where $\mathrm{V}_{0}$ is the cathode fall of potential, $\beta, v$, and $k$ are constants, $\gamma$ the ratio of the number of collisions in which corpuscles remain attached to the molecule to the total number of collisions, $\lambda$ the mean free path of a corpuscle, $c$ the thickness of the Crookes layer, and $e$ the charge on an ion.

It is difficult to understand the reason for many of the assumptions that are made in obtaining this formula For instance, it is supposed that the positive ions all traverse the Crookes layer, impinging on the cathode with a kinetic energy $e \mathrm{~V}_{0}$, a supposition which involves the assumption that they make no collisions with the molecules of the gas, although the length $c$ is much longer than the mean free path of the positive ions. This view is said to be supported by experiments which show that solid obstacles placed in the cathode dark space cast a shadow on the electrode. But it must be remembered that the solid obstacle which is placed near the cathode would presumably take up a potential less than the cathode fall of potential. The potential difference between the cathode and the obstacle in question would therefore be less than the minimum sparking potential, and no current could be maintained between the cathode and the obstacle. This should hold for any theory of the discharge, and the fact that the gas is not luminous in the space between the cathode and the obstacle supports equally all theories that account for the minimum sparking potential. 
With regard to the factor $\gamma$, the experimental determinations of currents between parallel plates show that the supposed effect which it is intended to represent either does not exist or else is so small as to be inappreciable. For let it be supposed that a certain number $m$ of the negative ions in going through the gas become attached to molecules and lose the property of ionizing the gas ; then if the conductivity be produced by ultra-violet light, as described in section 2 above, the equations for the determination of the quantity of electricity $q$ arriving at the positive electrode are $\frac{d n}{d x}=a n-\gamma n$ and $\frac{d m}{d x}=\gamma n$, where $n$ is the number of corpuscles which cross a plane at a distance $x$ from the negative electrode, and $d x$ the number of new ions generated in the distance $d x$, and $\gamma n d x$ the number of corpuscles that become attached to molecules in the distance $d x$. When $x=l$, the distance between the electrodes, $(m+n) e$ is the total charge $q$ arriving at the positive electrode, $e$ being the atomic charge.

The above equations give

$$
n=n_{0} \epsilon^{(a-\gamma) l} \text { and } m=n_{0} \frac{\gamma}{a-\gamma}\left(\epsilon^{(a-\gamma) l}-1\right)
$$

when $n_{0}$ corpuscles start from the negative electrode. Hence the quantity $q$ for the distance $l$ between the plates is given by the equation.

$$
\underline{e}=(m+n)=n_{0} \frac{a}{a-\gamma} \epsilon^{(a-\gamma) l}-\frac{n_{0} \gamma}{a-\gamma} .
$$

Now it has been found experimentally that the values of $q$ are given accurately by the formula $q=q_{0} \epsilon^{a l}$ even for the higher pressures and small electric forces where 
it might be expected that $\gamma$ would be large compared with $a$. The experimental evidence therefore shows that $\gamma=0$. If there is any adhesion of the negative corpuscles to molecules in the circumstances under consideration, the connection must be of a very loose and transitory character. ${ }^{1}$

In the 1906 edition of the treatise on "The Conduction of Electricity through Gases," Professor Sir J. J. Thomson gives another investigation of the sparking potential.

The sparking potential for parallel plate electrodes is obtained from the condition required to maintain a very small current which does not disturb the uniformity of the electric field, the lowering of the potential for large currents is attributed to the increase of force at the cathode, the effect of the positive ions in ionizing molecules of the gas is also taken into consideration, and for the larger distances between the plates this is regarded as the predominating effect of the positive ions. It will be noticed that it was on these principles, as has already been explained, ${ }^{2}$ that the theory which gives results in accurate agreement with experimental determinations was originally ${ }^{3}$ worked out.

The general expression for the potential is given by the equation ${ }^{4}$

$$
\frac{a-\gamma \epsilon}{\alpha-\gamma}^{(\alpha-\gamma) d}=k \alpha \frac{V e}{d}\left\{\frac{d \epsilon}{\alpha-\gamma-\beta}-\frac{(\alpha-\gamma-\beta) d}{\alpha-\gamma}-\frac{\epsilon^{(\alpha-\gamma-\beta) d}}{(\alpha-\gamma-\beta)^{2}}+\frac{1}{(\alpha-\gamma-\beta)^{2}}\right\}
$$

1 See section 7 above.

2 Sections 20, 21, and 22.

3 J. S. Townsend, Electrician, April, 1903; Philosophical Magazine, November, 1903, and March, 1905.

4 "The Conduction of Electricity through Gases," 1906 edition, pp. 494 and 495. 
in obtaining which it is supposed that ions are generated by the collisions of positive and negative ions with molecules of the gas, and also that negative ions are set free from the negative electrode when the positive ions collide with it. For large distances between the electrodes greater than the distance corresponding to the minimum sparking potential the condition for sparking becomes $\gamma=a \epsilon^{-a d}, \gamma$ representing the effect of the positive ions per centimetre in ionizing the gas. This equation is nearly the same as the equation $a=\beta \epsilon^{(a-\beta) \mathrm{S}}$ (section 23), since $\beta$ is small compared with $\alpha$, and it has been seen that this gives the correct expression for sparking.

With regard to the general expression for the condition for sparking given above, it is difficult to use the equation in order to make calculations of the potential, as the numerical values of the effects involved are not determined. From general principles, however, it appears obvious that it could not be very accurate for pressures or distances between the electrodes for which the product $p \times d$ is less than the critical value, as it fails to take into consideration processes of ionization which would account for experimental results with electrodes of various shapes and which would explain the variation of the potential with the current between parallel plates.

As has already been explained in section 28, the potential required to spark or maintain a current in a gas diminishes as the force becomes concentrated at the cathode, when the ions are produced by the action of positive and negative ions in the gas. In the general formula quoted above the system of ionization considered consists of the ions generated by positive and negative ions from molecules of the gas and in addition ions set 
free from the negative electrode when positive ions impinge on it. This additional process of ionization would obviously be greater when the force is concentrated near the cathode than when the larger force is at the anode. It is obvious, therefore, that when these three processes of ionization are acting alone, the potential required to give a spark or maintain a discharge should diminish when the force at the cathode increases, so that the results obtained from the general formula for sparking potentials could not be correct at the lower pressures.

In such cases it is necessary to introduce some important factor in addition to the direct effects of collisions, and a system which would account for the phenomena has been suggested in section 32 . It would be easy to find mathematical expressions, based on various assumptions, to express the sparking potentials under these conditions, but it is hardly desirable to make further investigations of sparking potentials at low pressures until some experimental evidence can be brought forward in support of the principles that are involved.

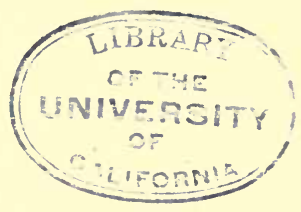




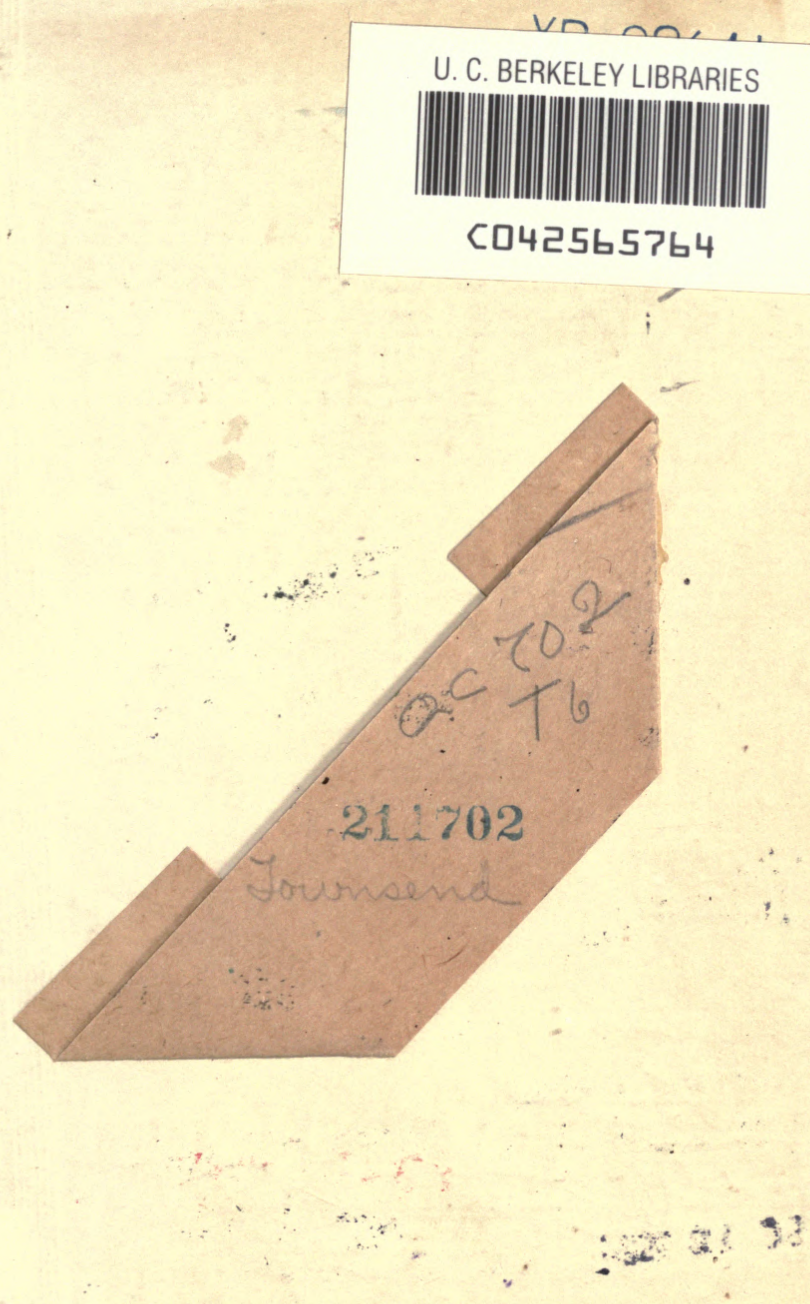
